\title{
SOME ISOPERIMETRIC INEQUALITIES WITH RESPECT TO MONOMIAL WEIGHTS
}

\author{
Angelo Alvino ${ }^{1}$, Friedemann Brock ${ }^{2}$, Francesco Chiacchio $^{1}$, \\ Anna Mercaldo ${ }^{1, *}$ and Maria Rosaria Posteraro ${ }^{1}$
}

\begin{abstract}
We solve a class of isoperimetric problems on $\mathbb{R}_{+}^{2}$ with respect to monomial weights. Let $\alpha$ and $\beta$ be real numbers such that $0 \leq \alpha<\beta+1, \beta \leq 2 \alpha$. We show that, among all smooth sets $\Omega$ in $\mathbb{R}_{+}^{2}$ with fixed weighted measure $\iint_{\Omega} y^{\beta} \mathrm{d} x \mathrm{~d} y$, the weighted perimeter $\int_{\partial \Omega} y^{\alpha} \mathrm{d} s$ achieves its minimum for a smooth set which is symmetric w.r.t. to the $y$-axis, and is explicitly given. Our results also imply an estimate of a weighted Cheeger constant and a bound for eigenvalues of some nonlinear problems.
\end{abstract}

Mathematics Subject Classification. 51M16, 46E35, 46E30, 35P15.

Received January 9, 2020. Accepted July 28, 2020.

\section{INTRODUCTION}

The last two decades have seen a growing interest in weighted isoperimetric problems in the Euclidean space. In many cases, volume and perimeter in those problems carried the same weight. Such a setting corresponds to manifolds with density, see for instance [5, 7, 8, 12, 15-18, 21, 36, 37, 41-43] and the references therein.

More recently, also problems with different weight functions for perimeter and volume were addressed, see for example $[2-4,6,23-26,33,38,39,45,46]$ and the references therein.

The minimizing sets for the isoperimetric problems are radial in all of the above-mentioned articles. There is only a sparse literature in the Euclidean setting with non-radial isoperimetric sets, see [1, 20, 27].

We wish to mention that isoperimetric problems w.r.t. non-Euclidean metrics, in particular in the framework of the Grushin space, have received a lot of attention recently, too, see for instance [28-30, 40] and references therein.

In this paper we deal with an isoperimetric problem in the upper half-plane, where the minimizers are not half balls, centered at the origin.

More precisely, we study the following problem:

Minimize $\int_{\partial \Omega} y^{\alpha} \mathrm{d} s$ among all smooth sets $\Omega \subset \mathbb{R}_{+}^{2}$ satisfying $\iint_{\Omega} y^{\beta} \mathrm{d} x \mathrm{~d} y=1$

Keywords and phrases: Isoperimetric inequality, weighted Cheeger set, eigenvalue problems.

${ }^{1}$ Università di Napoli Federico II, Dipartimento di Matematica e Applicazioni "R. Caccioppoli", Complesso Monte S. Angelo, via Cintia, 80126 Napoli, Italy.

${ }^{2}$ University of Rostock, Institute of Mathematics, 18057 Rostock, Ulmenstr. 69, Germany.

* Corresponding author: mercaldo@unina.it 
or equivalently

$$
\inf \left\{\frac{\int_{\partial \Omega} y^{\alpha} \mathrm{d} s}{\left[\iint_{\Omega} y^{\beta} \mathrm{d} x \mathrm{~d} y\right]^{(\alpha+1) /(\beta+2)}}: 0<\iint_{\Omega} y^{\beta} \mathrm{d} x \mathrm{~d} y<+\infty\right\}=: \mu(\alpha, \beta) .
$$

Our main result, proved in Section 2, is the following:

Theorem 1.1. Assume that

$$
0 \leq \alpha<\beta+1
$$

and

$$
\beta \leq 2 \alpha
$$

Then problem $(\mathbf{P})$ has a minimizer which is given by

$$
\begin{aligned}
\Omega^{\star} & :=\{(x, y):|x|<f(y), 0<y<1\}, \quad \text { where } \\
f(y) & :=\int_{y}^{1} \frac{t^{\beta-\alpha+1}}{\sqrt{1-t^{2(\beta-\alpha+1)}}} \mathrm{d} t, \quad(0<y<1) .
\end{aligned}
$$

Moreover, we have

$$
\mu(\alpha, \beta)=\gamma^{\frac{\alpha+1}{\beta+2}-1} \cdot\left[\frac{(\beta+1)(\beta+2)}{\alpha+1}\right]^{\frac{\alpha+1}{\beta+2}} \cdot\left[B\left(\frac{\alpha+1}{2 \gamma}, \frac{1}{2}\right)\right]^{\frac{\gamma}{\beta+2}},
$$

where $\gamma:=\beta+1-\alpha$ and $B$ denotes the Beta function. In particular,

$$
\mu(\alpha, 2 \alpha)=\sqrt{\frac{2 \pi(2 \alpha+1)}{\alpha+1}} .
$$

Remark 1.2. (a) First observe that $\Omega^{\star}$ is the half-circle when $\alpha=\beta$. Therefore Theorem 1.1 includes the result obtained by Maderna and Salsa in [37] (see also [11, 15]).

(b) Let $B_{1}^{+}:=\left\{(x, y) \in \mathbb{R}^{2}: x^{2}+y^{2}<1, y>0\right\}$. It is elementary to verify that,

1. if $\beta-\alpha<0$ then $\Omega \supseteq B_{1}^{+}$,

2. if $\beta-\alpha>0$ then $\Omega \subseteq B_{1}^{+}$,

3. if $\beta-\alpha=0$ then $\Omega \equiv B_{1}^{+}$; see Figure 1 .

Theorem 1.1 also allows to obtain a Faber-Krahn-type inequality for the so-called weighted Cheeger constant, and in turn a lower bound for the first eigenvalue for a degenerate elliptic operators. For similar results see also $[9,13,14,19,34,44,47]$.

We emphasize that our main result generalizes the isoperimetric inequality in the Grushin plane proved in [40] (see also [28-30]) which corresponds to Theorem 1.1 with $\alpha=0$ and $\beta \in(-1,0]$ (see Rem. 2.15 in Sect. 2).

In a forthcoming paper we will study the $N$-dimensional case, which has to be treated with a different approach.

Our paper is organized as follows. We state and prove the isoperimetric inequality, Theorem 1.1., in Section 2. We will also point out some relations to recent other literature, at the end of the section, see Remark 2.15. Further, we give two applications of Theorem 1.1., in Section 3. Namely, Theorem 3.1. gives an estimate for 


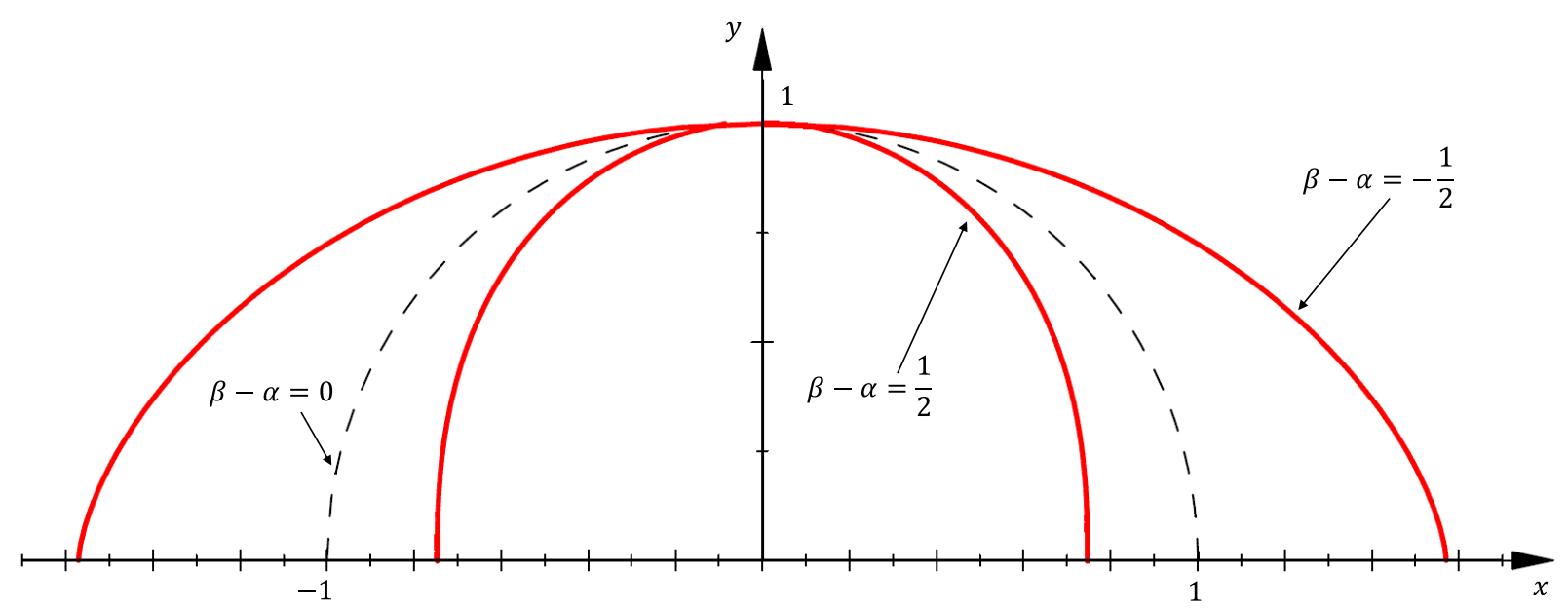

FiguRE 1. Isoperimetric sets for different values of $\alpha$ and $\beta$.

a weighted Cheeger constant while Theorem 3.2. provides a lower bound for the first eigenvalue of a class of non-linear degenerate weighted eigenvalue problems.

\section{ISOPERIMETRIC INEQUALITY IN THE UPPER HALF PLANE}

Let $\mathbb{R}_{+}^{2}:=\left\{(x, y) \in \mathbb{R}^{2}: y>0\right\}$. Throughout this paper, we assume that $\alpha, \beta \in \mathbb{R}$ and

$$
\beta+1>0 \text { and } \alpha \geq 0 .
$$

If $\Omega \subset \mathbb{R}_{+}^{2}$ is measurable, we set

$$
\begin{aligned}
\Omega(y) & :=\{x \in \mathbb{R}:(x, y) \in \Omega\}, \quad\left(y \in \mathbb{R}_{+}\right), \\
\Omega^{\prime} & :=\left\{y \in \mathbb{R}_{+}: \Omega(y) \neq \emptyset\right\} .
\end{aligned}
$$

Further, we define the weighted area of $\Omega$ by

$$
A_{\beta}(\Omega):=\iint_{\Omega} y^{\beta} \mathrm{d} x \mathrm{~d} y
$$

and the weighted relative perimeter of $\Omega$ as

$$
P_{\alpha}(\Omega):=\sup \left\{\iint_{\mathbb{R}_{+}^{2}} \operatorname{div}\left(y^{\alpha} \mathbf{v}\right) \mathrm{d} x d y: \mathbf{v} \in C_{0}^{1}\left(\mathbb{R}_{+}^{2}, \mathbb{R}^{2}\right),|\mathbf{v}| \leq 1 \text { in } \Omega\right\} .
$$

It is well-known that, if $\Omega$ is an open, rectifiable set, then the following equality holds

$$
P_{\alpha}(\Omega)=\int_{\partial \Omega \cap \mathbb{R}_{+}^{2}} y^{\alpha} \mathrm{d} \mathscr{H}_{1}
$$

( $\mathscr{H}_{1}$ denotes 1-dimensional Hausdorff-measure.) 
Remark 2.1. The following properties of the perimeter are well-known:

Let $\Omega$ be measurable with $0<A_{\beta}(\Omega)<+\infty$ and $P_{\alpha}(\Omega)<+\infty$.

Then there exists a sequence of open, rectifiable sets $\left\{\Omega_{n}\right\}$ with $\lim _{n \rightarrow \infty} A_{\beta}\left(\Omega \Delta \Omega_{n}\right)=0$ and

$$
P_{\alpha}(\Omega)=\lim _{n \rightarrow \infty} P_{\alpha}\left(\Omega_{n}\right) .
$$

Further, we have

$$
P_{\alpha}(\Omega) \leq \liminf _{n \rightarrow \infty} P_{\alpha}\left(\Omega_{n}\right)
$$

for any sequence of open, rectifiable sets $\left\{\Omega_{n}\right\}$ satisfying $\lim _{n \rightarrow \infty} A_{\beta}\left(\Omega \Delta \Omega_{n}\right)=0$.

We define the ratio

$$
\mathcal{R}_{\alpha, \beta}(\Omega):=\frac{P_{\alpha}(\Omega)}{\left[A_{\beta}(\Omega)\right]^{(\alpha+1) /(\beta+2)}}, \quad\left(0<A_{\beta}(\Omega)<+\infty\right) .
$$

Remark 2.2. We have $\mathcal{R}_{\alpha, \beta}(t \Omega)=\mathcal{R}_{\alpha, \beta}(\Omega)$ for every $t>0$.

We study the following isoperimetric problem:

$$
\inf \left\{\mathcal{R}_{\alpha, \beta}(\Omega): 0<A_{\beta}(\Omega)<+\infty\right\}=: \mu(\alpha, \beta) .
$$

Our first aim is to reduce the class of admissible sets in the isoperimetric problem (P).

Throughout our proofs let $C$ denote a generic constant which may vary from line but does not depend on the other parameters.

The first two Lemmata give necessary conditions for a minimizer to exist.

Lemma 2.3. If $\alpha>\beta+1$, then

$$
\mu(\alpha, \beta)=0,
$$

and $(\mathbf{P})$ has no minimizer.

Proof. Let $\Omega(t):=(0, t) \times(0,1),(t>0)$. Then

$$
\begin{aligned}
\int_{\partial \Omega(t) \cap \mathbb{R}_{+}^{2}} y^{\alpha} \mathrm{d} s & =t+2 \int_{0}^{t} y^{\alpha} d y=t+\frac{2}{\alpha+1}, \\
\iint_{\Omega(t)} y^{\beta} \mathrm{d} x \mathrm{~d} y & =\frac{t}{\beta+1} .
\end{aligned}
$$

Hence

$$
\mathcal{R}_{\alpha, \beta}(\Omega(t))=\frac{t+(2 /(\alpha+1))}{[t /(\beta+1)]^{(\alpha+1) /(\beta+2)}} \longrightarrow 0, \text { as } t \rightarrow+\infty,
$$

and the assertion follows. 
Lemma 2.4. Assume $\alpha<\beta+1$. Further, let $\Omega \subset \mathbb{R}_{+}^{2}$ be a nonempty, open and rectifiable set, which is not simply connected. Then there exists a nonempty, open and rectifiable set $U \subset \mathbb{R}_{+}^{2}$ which is simply connected, such that

$$
\mathcal{R}_{\alpha, \beta}(U)<\mathcal{R}_{\alpha, \beta}(\Omega) .
$$

Proof. (i) First assume that $\Omega$ is connected. Let $G$ be the unbounded component of $\mathbb{R}^{2} \backslash \bar{\Omega}$ and set $U:=\mathbb{R}^{2} \backslash \bar{G}$. Then $U$ is simply connected with $\Omega \subset U \subset \mathbb{R}_{+}^{2}$ and $\partial U \subset \partial \Omega$, so that (2.9) follows.

(ii) Next, let $\Omega=\cup_{k=1}^{m} \Omega_{k}$, with mutually disjoint, nonempty, open, connected and rectifiable sets $\Omega_{k},(k=$ $1, \ldots, m, m \geq 2)$. We set $\mathcal{R}_{\alpha, \beta}(\Omega)=: \lambda$. Let us assume that $\mathcal{R}_{\alpha, \beta}\left(\Omega_{k}\right) \geq \lambda$ for every $k \in\{1, \ldots, m\}$. Then we have, since $(\alpha+1) /(\beta+2)<1$,

$$
\begin{aligned}
P_{\alpha}(\Omega) & =\sum_{k=1}^{m} P_{\alpha}\left(\Omega_{k}\right) \geq \lambda \sum_{k=1}^{m}\left[A_{\beta}\left(\Omega_{k}\right)\right]^{(\alpha+1) /(\beta+2)} \\
& >\lambda\left[\sum_{k=1}^{m} A_{\beta}\left(\Omega_{k}\right)\right]^{(\alpha+1) /(\beta+2)}=\lambda\left[A_{\beta}(\Omega)\right]^{(\alpha+1) /(\beta+2)} \\
& =P_{\alpha}(\Omega)
\end{aligned}
$$

a contradiction. Hence there exists a number $k_{0} \in\{1, \ldots, m\}$ with $\mathcal{R}_{\alpha, \beta}\left(\Omega_{k_{0}}\right)<\lambda$. Then, repeating the argument of part (i), with $\Omega_{k_{0}}$ in place of $\Omega$, we again arrive at (2.9).

Lemma 2.5. There holds

$$
\mu(\beta+1, \beta)=\beta+1,
$$

but $\mathbf{( P )}$ has no open rectifiable minimizer.

Proof. With $\Omega(t)$ as in the proof of Lemma 2.3, we calculate $\mathcal{R}_{\beta+1, \beta}(\Omega(t))>\beta+1$ and

$$
\lim _{t \rightarrow \infty} \mathcal{R}_{\beta+1, \beta}(\Omega(t))=\beta+1 .
$$

Let $\Omega \subset \mathbb{R}_{+}^{2}$ be open, rectifiable and simply connected. Then $\partial \Omega$ is a closed Jordan curve $\mathcal{C}$ with counter-clockwise representation

$$
\mathcal{C}=\{(\xi(t), \eta(t)): 0 \leq t \leq a\}, \quad(a \in 0,+\infty),
$$

where $\xi, \eta \in C[0, a] \cap C^{0,1}(0, a), \xi(0)=\xi(a), \eta(0)=\eta(a)$, and $\left(\xi^{\prime}\right)^{2}+\left(\eta^{\prime}\right)^{2}>0$ on $[0, a]$. Using Green's Theorem we evaluate

$$
\begin{aligned}
A_{\beta}(\Omega) & =-\int_{\mathcal{C}} \frac{y^{\beta+1}}{\beta+1} \mathrm{~d} x=-\int_{0}^{a} \frac{[\eta(t)]^{\beta+1}}{\beta+1} \xi^{\prime}(t) \mathrm{d} t \\
& \leq \frac{1}{\beta+1} \int_{0}^{a}[\eta(t)]^{\beta+1} \sqrt{\left(\xi^{\prime}\right)^{2}+\left(\eta^{\prime}\right)^{2}} \mathrm{~d} t=\frac{1}{\beta+1} P_{\beta+1}(\Omega) .
\end{aligned}
$$

Equality in (2.12) can hold only if $\eta^{\prime} \equiv 0$ and $\xi^{\prime} \leq 0$ on $\partial \Omega \cap \mathbb{R}_{+}^{2}$, that is, if $\partial \Omega \cap \mathbb{R}_{+}^{2}$ is a single straight segment which is parallel to the $x$-axis. But this is impossible. Hence we find that

$$
P_{\beta+1}(\Omega)>(\beta+1) A_{\beta}(\Omega) .
$$


To show the assertion in the general case, we proceed similarly as in the proof of Lemma 2.4:

Assume first that $\Omega$ is connected and define the sets $G$ and $U$ as in the last proof. Using (2.13), with $U$ in place of $\Omega$, we obtain

$$
\begin{aligned}
P_{\beta+1}(\Omega) & \geq P_{\beta+1}(U) \\
& >(\beta+1) A_{\beta}(U) \geq(\beta+1) A_{\beta}(\Omega) .
\end{aligned}
$$

Finally, let $\Omega$ be open and rectifiable. Then $\Omega=\cup_{k=1}^{m} \Omega_{k}$, with mutually disjoint, connected sets $\Omega_{k},(k=$ $1, \ldots, m)$. Then $(2.14)$ yields

$$
\begin{aligned}
P_{\beta+1}(\Omega) & =\sum_{k=1}^{m} P_{\beta+1}\left(\Omega_{k}\right) \\
& >(\beta+1) \sum_{k=1}^{m} A_{\beta}\left(\Omega_{k}\right)=(\beta+1) A_{\beta}(\Omega) .
\end{aligned}
$$

Now the assertion follows from (2.15) and (2.11).

Lemma 2.6. Let $2 \alpha<\beta$. Then (2.8) holds and (P) has no minimizer.

Proof. Let $z(t):=(0, t),(t \geq 2)$. Then we have for all $t \geq 2$,

$$
\begin{gathered}
\int_{\partial B_{1}(z(t))} y^{\alpha} \mathrm{d} s \leq C t^{\alpha} \text { and } \\
\iint_{B_{1}(z(t))} y^{\beta} \mathrm{d} x \mathrm{~d} y \geq C t^{\beta} .
\end{gathered}
$$

This implies

$$
\mathcal{R}_{\alpha, \beta}\left(B_{1}(z(t))\right) \leq C t^{\alpha-\beta(1+\alpha) /(2+\beta)} \longrightarrow 0, \text { as } t \rightarrow+\infty,
$$

and the assertion follows.

Next we recall the definition of the Steiner symmetrization w.r.t. the $x$-variable. If $\Omega$ is measurable, we set

$$
S(\Omega):=\left\{(x, y): x \in S(\Omega(y)), y \in \Omega^{\prime}\right\}
$$

where $\Omega(y), \Omega^{\prime}$ are defined in $(2.2)$ and

$$
S(\Omega(y)):= \begin{cases}\left(-\frac{1}{2} \mathscr{L}^{1}(\Omega(y)),+\frac{1}{2} \mathscr{L}^{1}(\Omega(y))\right) & \text { if } 0<\mathscr{L}^{1}(\Omega(y))<+\infty \\ \emptyset & \text { if } \mathscr{L}^{1}(\Omega(y))=0 \\ \mathbb{R} & \text { if } \mathscr{L}^{1}(\Omega(y))+\infty\end{cases}
$$

Note that $S(\Omega)(y)$ is a symmetric interval with $\mathscr{L}^{1}(S(\Omega)(y))=\mathscr{L}^{1}(\Omega(y))$.

Since the weight functions in the functionals $P_{\alpha}$ and $A_{\beta}$ do not depend on $x$, we have the following well-known properties, see ([32], Prop. 3).

Lemma 2.7. Let $\Omega \subset \mathbb{R}_{+}^{2}$ be measurable. Then

$$
P_{\alpha}(\Omega) \geq P_{\alpha}(S(\Omega)) \quad \text { and }
$$




$$
A_{\beta}(\Omega)=A_{\beta}(S(\Omega))
$$

For nonempty open sets $\Omega$ with $\Omega=S(\Omega)$ we set $\Omega_{t}:=\{(x, y) \in \Omega: y>t\}$ and

$$
\begin{aligned}
& y^{+}:=\inf \left\{t \geq 0: A_{\beta}\left(\Omega_{t}\right)=0\right\}, \\
& y^{-}:=\sup \left\{t \geq 0: A_{\beta}\left(\Omega \backslash \Omega_{t}\right)=0\right\} .
\end{aligned}
$$

Remark 2.8. Assume that $\Omega \subset \mathbb{R}_{+}^{2}$ is a bounded, open and rectifiable set with $0<A_{\beta}(\Omega)<+\infty$ and $\Omega=S(\Omega)$. Then it has the following representation,

$$
\begin{aligned}
\Omega= & \left\{(x, y):|x|<f(y), y^{-}<y<y^{+}\right\}, \quad \text { where } \\
& f:\left(y^{-}, y^{+}\right) \rightarrow(0,+\infty) \text { is lower semi-continuous. }
\end{aligned}
$$

Lemma 2.9. Let $\Omega$ be a nonempty, bounded, open and rectifiable set with $\Omega=S(\Omega)$. Then we have

$$
\begin{aligned}
& P_{\alpha}(\Omega) \geq \frac{2}{\alpha+1}\left(\left(y^{+}\right)^{\alpha+1}-\left(y^{-}\right)^{\alpha+1}\right) \quad \text { and } \\
& P_{\alpha}(\Omega) \geq 2 y^{\alpha} f(y) \quad \forall y \in\left(y^{-}, y^{+}\right)
\end{aligned}
$$

where $f$ is given by (2.21).

Proof. Assume first that $\Omega$ is represented by (2.21) where

$$
f \in C^{1}\left[y^{-}, y^{+}\right] \text {and } f\left(y^{-}\right)=f\left(y^{+}\right)=0 .
$$

Then we have for every $y \in\left(y^{-}, y^{+}\right)$,

$$
\begin{aligned}
P_{\alpha}(\Omega) & =2 \int_{y^{-}}^{y^{+}} t^{\alpha} \sqrt{1+\left(f^{\prime}(t)\right)^{2}} \mathrm{~d} t \geq 2 \int_{y}^{y^{+}} t^{\alpha} \sqrt{1+\left(f^{\prime}(t)\right)^{2}} \mathrm{~d} t \\
& \geq 2 y^{\alpha} \int_{y}^{y^{+}}\left|f^{\prime}(t)\right| \mathrm{d} t \geq 2 y^{\alpha} f(y) .
\end{aligned}
$$

Furthermore, there holds

$$
\begin{aligned}
P_{\alpha}(\Omega) & =2 \int_{y^{-}}^{y^{+}} t^{\alpha} \sqrt{1+\left(f^{\prime}(t)\right)^{2}} \mathrm{~d} t \geq 2 \int_{y^{-}}^{y^{+}} t^{\alpha} \mathrm{d} t \\
& =\frac{2}{\alpha+1}\left(\left(y^{+}\right)^{\alpha+1}-\left(y^{-}\right)^{\alpha+1}\right) .
\end{aligned}
$$

In the general case the assertions follow from these calculations by approximation with sets $\Omega$ of the type given by $(2.21),(2.24)$.

Lemma 2.10. Assume that

$$
\begin{aligned}
& \alpha<\beta+1, \quad \text { and } \\
& \beta<2 \alpha,
\end{aligned}
$$


and let $\Omega$ be a bounded, open and rectifiable set with $A_{\beta}(\Omega)=1, \Omega=S(\Omega)$ and $P_{\alpha}(\Omega)<\mu(\alpha, \beta)+1$. Then there exist positive numbers $C_{1}$ and $C_{2}$ which depend only on $\alpha$ and $\beta$ such that

$$
C_{1} \geq y^{+} \quad \text { and } \quad y^{+}-y^{-} \geq C_{2} .
$$

Proof. By (2.22) and (2.23) we have

$$
\begin{aligned}
\frac{1}{2}(\mu(\alpha, \beta)+1)(\alpha+1) & \geq\left(y^{+}\right)^{\alpha+1}-\left(y^{-}\right)^{\alpha+1} \text { and } \\
\frac{\mu(\alpha, \beta)+1}{2} & \geq y^{\alpha} f(y) \quad \forall y \in\left(y^{-}, y^{+}\right) .
\end{aligned}
$$

It follows that

$$
\begin{aligned}
1 & =A_{\beta}(\Omega)=2 \int_{y^{-}}^{y^{+}} y^{\beta} f(y) d y \\
& \leq(\mu(\alpha, \beta)+1) \int_{y^{-}}^{y^{+}} y^{\beta-\alpha} d y=\frac{\mu(\alpha, \beta)+1}{\beta+1-\alpha}\left(\left(y^{+}\right)^{\beta+1-\alpha}-\left(y^{-}\right)^{\beta+1-\alpha}\right) .
\end{aligned}
$$

Setting $z:=\frac{y^{-}}{y^{+}}(\in[0,1))$, we obtain from (2.28) and (2.30),

$$
\begin{aligned}
\left(y^{+}\right)^{\alpha+1} & \leq \frac{(\mu(\alpha, \beta)+1)(\alpha+1)}{2\left(1-z^{\alpha+1}\right)} \text { and } \\
\left(y^{+}\right)^{\beta+1-\alpha} & \geq \frac{\beta+1-\alpha}{(\mu(\alpha, \beta)+1)\left(1-z^{\beta+1-\alpha}\right)},
\end{aligned}
$$

which implies that

$$
f(z):=\frac{\left(1-z^{\alpha+1}\right)^{(\beta+1-\alpha) /(\alpha+1)}}{1-z^{\beta+1-\alpha}} \leq C,
$$

with a constant $C$ which depends only on $\alpha$ and $\beta$. By (2.26) we have that

$$
\lim _{z \rightarrow 1^{-}} f(z)=+\infty \text {. }
$$

Hence it follows that

$$
z=\frac{y^{-}}{y^{+}} \leq 1-\delta \text { for some } \delta \in(0,1) .
$$

Using (2.31) and (2.32) this leads to (2.27).

Lemma 2.11. Assume (2.25) and (2.26). Then problem (P) has a minimizer $\Omega^{\star}$ which is symmetric w.r.t. the $y$-axis.

Proof. We proceed in 4 steps.

Step 1: A minimizing sequence:

Let $\left\{\Omega_{n}\right\}$ a minimizing sequence, that is, $\lim _{n \rightarrow \infty} \mathcal{R}_{\alpha, \beta}\left(\Omega_{n}\right)=\mu(\alpha, \beta)$. In view of the Remarks 2.1 and 2.2 and the Lemmata 2.4 and 2.9 we may assume that $\Omega_{n}$ is open, simply connected and rectifiable with $\Omega_{n}=S\left(\Omega_{n}\right)$, 
$A_{\beta}\left(\Omega_{n}\right)=1$ and $P_{\alpha}\left(\Omega_{n}\right) \leq \mu(\alpha, \beta)+\frac{1}{n},(n \in \mathbb{N})$.

Step 2: Parametrization of $\partial \Omega_{n}$ :

Denote $\mathcal{C}_{n}:=\overline{\partial \Omega_{n} \cap\{(x, y): x>0, y>0\}}$. By a standard approximation procedure we may assume that $\mathcal{C}_{n}$ is a simple smooth curve with

$$
\mathcal{C}_{n}=\left\{\left(x_{n}(s), y_{n}(s)\right): s \in\left[0, L_{n}\right]\right\}
$$

where $s$ denotes the usual arclength parameter, $L_{n} \in(0,+\infty), x_{n}, y_{n} \in C\left[0, L_{n}\right] \cap C^{0,1}\left(0, L_{n}\right), x_{n}(s)>0$ and $y_{n}(s)>0$ for every $s \in\left(0, L_{n}\right),(n \in \mathbb{N})$. We orientate $\mathcal{C}_{n}$ in such a way that the mapping $s \mapsto y_{n}(s)$ is nonincreasing and $x_{n}(0)=0$. Setting $y_{n}(0)=: y_{n}^{+}$and $y_{n}\left(L_{n}\right)=: y_{n}^{-}$, we have by Lemma 2.9,

$$
C_{1} \geq y_{n}^{+} \quad \text { and } y_{n}^{+}-y_{n}^{-} \geq C_{2},
$$

where $C_{1}$ and $C_{2}$ do not depend on $n$. Note that $x_{n}\left(L_{n}\right)=0$ in case that $y_{n}^{-}>0$. Further, Lemma 2.9, (2.23) shows that

$$
\mu(\alpha, \beta)+\frac{1}{n} \geq 2 y_{n}(s)^{\alpha} x_{n}(s), \quad \forall s \in\left[0, L_{n}\right)
$$

For our purposes it will be convenient to work with another parametrization of $\mathcal{C}_{n}$ : We set

$$
\begin{aligned}
\varphi_{n}(s) & :=\frac{2}{P_{\alpha}\left(\Omega_{n}\right)} \int_{0}^{s} y_{n}^{\alpha}(t) \mathrm{d} t, \quad \text { and } \\
X_{n}\left(\varphi_{n}(s)\right) & :=x_{n}(s), Y_{n}\left(\varphi_{n}(s)\right):=y_{n}(s), \quad\left(s \in\left[0, L_{n}\right]\right) .
\end{aligned}
$$

Then $X_{n}, Y_{n} \in C[0,1] \cap C^{0,1}(0,1)$, and we evaluate

$$
\begin{aligned}
1=A_{\beta}\left(\Omega_{n}\right) & =\frac{2}{\beta+1} \int_{0}^{1} Y_{n}^{\beta+1}(\sigma) X_{n}^{\prime}(\sigma) \mathrm{d} \sigma=-2 \int_{0}^{1} Y_{n}^{\beta}(\sigma) Y_{n}^{\prime}(\sigma) X_{n}(\sigma) \mathrm{d} \sigma, \\
P_{\alpha}\left(\Omega_{n}\right) & =2 \int_{0}^{1} Y_{n}^{\alpha}(\sigma) \sqrt{\left(X_{n}^{\prime}(\sigma)\right)^{2}+\left(Y_{n}^{\prime}(\sigma)\right)^{2}} \mathrm{~d} \sigma, \\
\frac{\mathrm{d}}{\mathrm{d} \sigma} X_{n}(\sigma) & =\frac{P_{\alpha}\left(\Omega_{n}\right)}{2}\left(y_{n}(s)\right)^{-\alpha} x_{n}^{\prime}(s), \\
\frac{\mathrm{d}}{\mathrm{d} \sigma} Y_{n}(\sigma) & =\frac{P_{\alpha}\left(\Omega_{n}\right)}{2}\left(y_{n}(s)\right)^{-\alpha} y_{n}^{\prime}(s), \quad\left(\sigma=\varphi_{n}(s)\right) .
\end{aligned}
$$

Step 3: Limit of the minimizing sequence:

Since $\left(x_{n}^{\prime}(s)\right)^{2}+\left(y_{n}^{\prime}(s)\right)^{2} \equiv 1$, we obtain from (2.34) and (2.39) that the family $\left\{Y_{n}^{\alpha+1}\right\}$ is equibounded and uniformly Lipschitz continuous on $(0,1)$. Hence there is a function $Y \in C[0,1]$ with $Y^{\alpha+1} \in C^{0,1}(0,1)$ such that, up to a subsequence,

$$
Y_{n}^{\alpha+1} \longrightarrow Y^{\alpha+1} \quad \text { uniformly on }[0,1]
$$

Moreover, setting $Y(0)=: y^{+}, Y(1)=: y^{-}$, the bounds (2.27) are in place and $\sigma \mapsto Y(\sigma)$ is nonincreasing.

Let

$$
\sigma_{0}:=\sup \{\sigma \in(0,1]: Y(\sigma)>0\}
$$


Then from (2.35) and (2.38) we obtain that the families $\left\{X_{n}\right\}$ and $\left\{\mathrm{d} X_{n} / \mathrm{d} \sigma\right\}$ are equibounded on every closed subset of $\left[0, \sigma_{0}\right)$. Hence there exists a function $X \in C\left[0, \sigma_{0}\right)$ which is locally Lipschitz continuous on $\left[0, \sigma_{0}\right)$, such that, up to a subsequence,

$$
X_{n} \rightarrow X \quad \text { uniformly on closed subsets of }\left[0, \sigma_{0}\right)
$$

Moreover, from (2.35) and (2.38) we find that

$$
\begin{aligned}
\mu(\alpha, \beta) & \geq 2(Y(\sigma))^{\alpha} X(\sigma) \quad \text { and } \\
\mu(\alpha, \beta)+1 & \geq 2(Y(\sigma))^{\alpha} X^{\prime}(\sigma), \quad\left(\sigma \in\left[0, \sigma_{0}\right)\right) .
\end{aligned}
$$

Let $\Omega$ be the set in $\mathbb{R}_{+}^{2}$ with $\Omega=S(\Omega)$ such that $\partial \Omega \cap\{(x, y): x>0, y>0\}$ is represented by the pair of functions

$$
X(\sigma), Y(\sigma): \quad \sigma \in\left[0, \sigma_{0}\right)
$$

In view of (2.43) we have that

$$
A_{\beta}(\Omega)=\frac{2}{\beta+1} \int_{0}^{\sigma_{0}} Y^{\beta+1} X^{\prime} \mathrm{d} \sigma
$$

Step 4: A minimizing set:

We prove that

$$
A_{\beta}(\Omega)=1, \quad P_{\alpha}(\Omega)=\mu(\alpha, \beta) .
$$

In order to prove the first equality, since $A_{\beta}\left(\Omega_{n}\right)=1$, we prove that

$$
\lim _{n \rightarrow+\infty} A_{\beta}\left(\Omega_{n}\right)=A_{\beta}(\Omega) .
$$

Fix some $\delta \in\left(0, \sigma_{0}\right)$. Then (2.38), (2.39), (2.43), (2.40) and (2.41) yield

$$
\begin{aligned}
& \left|\int_{0}^{\sigma_{0}-\delta} Y_{n}^{\beta+1} X_{n}^{\prime} \mathrm{d} \sigma-\int_{0}^{\sigma_{0}-\delta} Y^{\beta+1} X^{\prime} \mathrm{d} \sigma\right| \\
\leq & \left|\int_{0}^{\sigma_{0}-\delta} Y_{n}^{\beta+1}\left(X_{n}^{\prime}-X^{\prime}\right) \mathrm{d} \sigma\right|+\int_{0}^{\sigma_{0}-\delta}\left|Y_{n}^{\beta+1}-Y^{\beta+1}\right|\left|X^{\prime}\right| \mathrm{d} \sigma \\
\leq & \left.Y_{n}^{\beta+1}\left|X_{n}-X\right|\right|_{0} ^{\sigma_{0}-\delta}+(\beta+1) \int_{0}^{\sigma_{0}-\delta} Y_{n}^{\beta}\left|Y_{n}^{\prime}\right|\left|X_{n}-X\right| \mathrm{d} \sigma \\
& +\int_{0}^{\sigma_{0}-\delta}\left|Y_{n}^{\beta+1}-Y^{\beta+1}\right|\left|X^{\prime}\right| \mathrm{d} \sigma \quad \longrightarrow 0, \quad \text { as } n \rightarrow \infty .
\end{aligned}
$$


Further, (2.38) and (2.43) give

$$
\begin{aligned}
\lim _{t \rightarrow 0} \int_{\sigma_{0}-t}^{\sigma_{0}}\left(Y_{n}\right)^{\beta+1} X_{n}^{\prime} \mathrm{d} \sigma & =0, \quad \text { uniformly for all } n \in \mathbb{N}, \text { and } \\
\lim _{t \rightarrow 0} \int_{\sigma_{0}-t}^{\sigma_{0}} Y^{\beta+1} X^{\prime} \mathrm{d} \sigma & =0 .
\end{aligned}
$$

Now (2.48), (2.49), (2.47) and (2.44) yield (2.46) and therefore the first of the equalities in (2.45).

Now we prove the second inequality in (2.45). With $\delta$ as above we also have

$$
\begin{aligned}
P_{\alpha}\left(\Omega_{n}\right) & =2 \int_{0}^{1} Y_{n}^{\alpha} \sqrt{\left(X_{n}^{\prime}\right)^{2}+\left(Y_{n}^{\prime}\right)^{2}} \mathrm{~d} \sigma \\
& \geq \int_{0}^{\sigma_{0}-\delta}\left(Y_{n}^{\alpha}-Y^{\alpha}\right) \sqrt{\left(X_{n}^{\prime}\right)^{2}+\left(Y_{n}^{\prime}\right)^{2}} \mathrm{~d} \sigma+\int_{0}^{\sigma_{0}-\delta} Y^{\alpha} \sqrt{\left(X_{n}^{\prime}\right)^{2}+\left(Y_{n}^{\prime}\right)^{2}} \mathrm{~d} \sigma \\
& =: I_{n, \delta}^{1}+I_{n, \delta}^{2} .
\end{aligned}
$$

In view of (2.40) and (2.41) it follows that $\lim _{n \rightarrow \infty} I_{n, \delta}^{1}=0$.

On the other hand, we have

$$
\liminf _{n \rightarrow \infty} I_{n, \delta}^{2} \geq \int_{0}^{\sigma_{0}-\delta} Y^{\alpha} \sqrt{\left(X^{\prime}\right)^{2}+\left(Y^{\prime}\right)^{2}} \mathrm{~d} \sigma
$$

Define

$$
S:=\{\sigma \in(0,1): X(\sigma)>0, Y(\sigma)>0\}
$$

Letting $\delta \rightarrow 0$ we obtain

$$
\begin{aligned}
\mu(\alpha, \beta) & \geq \liminf _{n \rightarrow \infty} P_{\alpha}\left(\Omega_{n}\right) \geq \int_{0}^{\sigma_{0}} Y^{\alpha} \sqrt{\left(X^{\prime}\right)^{2}+\left(Y^{\prime}\right)^{2}} \mathrm{~d} \sigma \\
& \geq \int_{S} Y^{\alpha} \sqrt{\left(X^{\prime}\right)^{2}+\left(Y^{\prime}\right)^{2}} \mathrm{~d} \sigma \\
& =P_{\alpha}(\Omega) \geq \mu(\alpha, \beta) .
\end{aligned}
$$

Hence $\Omega$ is a minimizing set.

Note that $\Omega$ must be simply connected in view of Lemma 2.4, which implies that there is a number $\sigma_{1} \in\left[0, \sigma_{0}\right)$ such that

$$
S=\left(\sigma_{1}, \sigma_{0}\right)
$$

This finishes the proof of Lemma 2.11 .

Next we obtain differential equations for the functions $X$ and $Y$ in the proof in Lemma 2.11. 
Lemma 2.12. Assume (2.25) and (2.26). Then the minimizer $\Omega$ obtained in Lemma 2.8 is bounded, and its boundary given parametrically by

$$
\begin{aligned}
& \partial \Omega \cap\left\{(x, y) \in \mathbb{R}^{2}: x>0, y>0\right\} \\
= & \{(x(s), y(s)): s \in(0, L)\}, \quad(s: \text { arclength }),
\end{aligned}
$$

where the functions $x, y \in C^{2}[0, L]$ satisfy the following equations:

$$
\begin{aligned}
& -\left(y^{\alpha} y^{\prime}\right)^{\prime}+\alpha y^{\alpha-1}=\lambda(\beta+1) y^{\beta} x^{\prime}, \\
& -\left(y^{\alpha} x^{\prime}\right)^{\prime}=-\lambda\left(y^{\beta+1}\right)^{\prime},
\end{aligned}
$$

together with the boundary conditions

$$
\begin{gathered}
x(0)=0, y(0)=y^{+}, y^{\prime}(0)=0, \\
y(L)=y^{-}, \quad \text { and either } \\
(i) y^{-}>0, y^{\prime}(L)=0, \quad \text { or } \\
\text { (ii) } y^{-}=0, \lim _{s \nearrow L} y^{\alpha}(s) x^{\prime}(s)=0,
\end{gathered}
$$

for some numbers $\lambda>0$ and $0 \leq y^{-}<y^{+}$. Finally, the curve (2.51) is strictly convex.

Proof. We proceed in 4 steps.

Step 1: Euler equations:

After a rescaling the parameter $\sigma$, we see that the functions $X(\sigma)$ and $Y(\sigma)$ in the previous proof annihilate the first variation of the functional

$$
\int_{0}^{1} Y^{\alpha} \sqrt{\left(X^{\prime}\right)^{2}+\left(Y^{\prime}\right)^{2}} \mathrm{~d} \sigma
$$

under the constraint

$$
\int_{0}^{1} Y^{\beta+1} X^{\prime} \mathrm{d} \sigma=\text { const. }(>0) .
$$

Hence $X$ and $Y$ satisfy the Euler equations

$$
\begin{aligned}
& -\frac{\mathrm{d}}{\mathrm{d} \sigma}\left(\frac{Y^{\alpha} Y^{\prime}}{\sqrt{\left(X^{\prime}\right)^{2}+\left(Y^{\prime}\right)^{2}}}\right)+\alpha Y^{\alpha-1} \sqrt{\left(X^{\prime}\right)^{2}+\left(Y^{\prime}\right)^{2}}=\lambda(\beta+1) Y^{\beta} X^{\prime}, \\
& -\frac{\mathrm{d}}{\mathrm{d} \sigma}\left(\frac{Y^{\alpha} X^{\prime}}{\sqrt{\left(X^{\prime}\right)^{2}+\left(Y^{\prime}\right)^{2}}}\right)=-\lambda \frac{\mathrm{d}}{\mathrm{d} \sigma}\left(Y^{\beta+1}\right),
\end{aligned}
$$

where $\lambda$ is a Lagrangian multiplier, and

$$
X(\sigma)>0, Y(\sigma)>0 .
$$

In addition, the following boundary conditions are satisfied:

$$
X(0)=0, Y(0)=: y^{+}>0, Y^{\prime}(0)=0,
$$




$$
\begin{aligned}
& \text { if } Y(1)=0 \text { and } \lim _{\sigma \rightarrow 1} X(\sigma) \text { exists, then } \lim _{\sigma \rightarrow 1} Y^{\alpha}(\sigma) X^{\prime}(\sigma)=0 \text {, } \\
& \text { if } Y(1)=: y^{-}>0 \text {, then } X(1)=0 \text { and } X^{\prime}(1)=0 .
\end{aligned}
$$

\section{Step 2: Boundedness:}

It will be more convenient to rewrite the above conditions in terms of the arclength parameter $s$ : Set

$$
\begin{aligned}
& s \equiv \psi(\sigma):=\int_{0}^{\sigma} \sqrt{\left(X^{\prime}(t)\right)^{2}+\left(Y^{\prime}(t)\right)^{2}} \mathrm{~d} t, \\
& x(s):=X(\psi(s)), y(s):=Y(\psi(s)), \quad(s \in(0, L)),
\end{aligned}
$$

where $L \in(0,+\infty]$. Then we have $\left(x^{\prime}(s)\right)^{2}+\left(y^{\prime}(s)\right)^{2}=1$ so that $(2.57),(2.58)$ yield the system of equations (2.52), (2.53).

Now we prove that $x, y$ are smooth functions. Firstly let us prove that $x, y \in C^{1}(10, L[)$. Indeed, by the previous step $x, y$ are Lipschitz continuous functions, i.e. $x, y \in W_{l o c}^{1, \infty}(] 0, L[)$. By Lemma 8.1, p. 204 of [10], there exists a constant $d$ such that

$$
\left.y^{\alpha} x^{\prime}-\lambda y^{\beta+1}=d, \quad \text { a.e. in }\right] 0, L[,
$$

or equivalently, since $y(\sigma)>0$ in $] 0, L[$,

$$
\left.x^{\prime}=\lambda y^{\beta+1-\alpha}+\mathrm{d} y^{-\alpha}, \quad \text { a.e. in }\right] 0, L[.
$$

Since $y^{\alpha} \in C(] 0, L[)$, we deduce that $x^{\prime} \in C(] 0, L[)$, that is

$$
x \in C^{1}(] 0, L[) .
$$

Now by $(2.53)$

$$
-\left(y^{\alpha} y^{\prime}\right)^{\prime}=\lambda(\beta+1) y^{\beta} x^{\prime}-\alpha y^{\alpha-1} \in C(] 0, L[),
$$

and, since $y^{\alpha+1}, y$ are locally Lipschitz continuous functions by the previous step, we conclude that

$$
y^{\alpha} y^{\prime} \in W_{l o c}^{1, \infty}(] 0, L[) .
$$

By Remark 6, p. 204 of [10], we conclude that

$$
y^{\alpha} y^{\prime} \in C_{l o c}^{1}(] 0, L[) .
$$

Since $y^{\alpha} \in C(] 0, L[)$, we deduce $y^{\prime} \in C(] 0, L[)$ or, equivalently

$$
y \in C^{1}(] 0, L[) .
$$

By (2.63) this implies that $x^{\prime} \in C^{1}(] 0, L[)$, i.e. $x \in C^{2}(] 0, L[)$.

Moreover by $(2.64),-\left(y^{\alpha} y^{\prime}\right)^{\prime} \in C^{1}(] 0, L[)$ since $y^{\alpha} \in C^{1}(] 0, L[)$, this means that $y^{\prime} \in C^{1}(] 0, L[)$. In a similar way we deduce that also $y \in C^{2}(] 0, L[)$. By repeating this argument we conclude that $x, y$ are smooth functions on $] 0, L[$.

Integrating (2.53) we obtain

$$
y^{\alpha} x^{\prime}=\lambda y^{\beta+1}+d,
$$


for some $d \in \mathbb{R}$.

Assume first that $x(s)$ is unbounded. Then $L=+\infty$, and in view of $(2.42)$ we have that $\lim _{s \rightarrow \infty} y(s)=0$. If $\alpha=0$, then this would imply $P_{0}(\Omega)=+\infty$, which is impossible. Hence we may restrict ourselves to the case $\alpha>0$.

There is a sequence $s_{n} \rightarrow+\infty$ such that $\lim _{n \rightarrow \infty} x^{\prime}\left(s_{n}\right)=1$. Using $s=s_{n}$ in (2.65) and passing to the limit $n \rightarrow \infty$ gives $d=0$. Plugging this into (2.52), we find

$$
-\left(y^{\alpha} y^{\prime}\right)^{\prime}+\alpha y^{\alpha-1}=\lambda^{2}(\beta+1) y^{2 \beta+1-\alpha} .
$$

Multiplying (2.66) with $y^{\alpha} y^{\prime}$ and integrating, we obtain

$$
-y^{2 \alpha}\left(y^{\prime}\right)^{2}+\alpha y^{2 \alpha}=\lambda^{2}(\beta+1) y^{2 \beta+2}+D,
$$

or equivalently,

$$
-\left(y^{\prime}\right)^{2}+\alpha=\lambda^{2}(\beta+1) y^{2 \beta+2-2 \alpha}+D y^{-2 \alpha},
$$

for some $D \in \mathbb{R}$. Using $s=s_{n}$ in (2.67) and taking into account that $\lim _{n \rightarrow+\infty} y^{\prime}\left(s_{n}\right)=0, \beta+1-\alpha>0$ and $\alpha>0$, we arrive again at a contradiction. Hence $x(s)$ is bounded, and we deduce $L<+\infty$.

Finally we can apply ([10], Thm. 8.2, p. 204) and we deduce the existence of continuous representatives on $[0, L] \bar{x}$ and $\bar{y}$ of $x$ and $y$ respectively such that

$$
\bar{x}, \bar{y} \in C^{2}([0, L]) \quad \text { and } \quad x=\bar{x}, y=\bar{y} \text { a.e. in }[0, L] .
$$

We identify $x$ and $y$ with their continuous representative $\bar{x}, \bar{y}$.

Therefore we deduce that $x, y \in C^{2}[0, L]$ and the boundary conditions (2.54)-(2.56).

Step 3: $\lambda$ is positive:

Multiplying (2.52) with $y$ and integrating from $s=0$ to $s=L$ gives

$$
-\int_{0}^{L}\left(y^{\alpha} y^{\prime}\right)^{\prime} y \mathrm{~d} s+\alpha \int_{0}^{L} y^{\alpha} \mathrm{d} s=\lambda(\beta+1) \int_{0}^{L} y^{\beta+1} x^{\prime} \mathrm{d} s .
$$

Using integration by parts this yields

$$
-\left.y^{\alpha+1} y^{\prime}\right|_{0} ^{L}+\int_{0}^{L} y^{\alpha}\left(\left(y^{\prime}\right)^{2}+\alpha\right) \mathrm{d} s=\left.\lambda(\beta+1) y^{\beta+1} x\right|_{0} ^{L}+\lambda(\beta+1)^{2} \int_{0}^{L} y^{\beta}\left(-y^{\prime}\right) x \mathrm{~d} s .
$$

The first two boundary terms in this identity vanish due to the boundary conditions (2.54)-(2.56) and the two integrals are positive since $y^{\prime} \leq 0$ and $y^{\prime} \not \equiv 0$. It follows that

$$
\lambda>0 .
$$

Now, considering equation (2.65) at $s=L$ and taking into account the boundary conditions (2.56), we find

$$
0 \geq \lambda\left(y^{-}\right)^{\beta+1}+d
$$

which implies that

$$
d \leq 0
$$


Step 4: Strict convexity:

From (2.52) and (2.53) we obtain

$$
\begin{aligned}
& x^{\prime \prime}=\lambda(\beta+1) y^{\beta-\alpha} y^{\prime}-\frac{\alpha}{y} x^{\prime} y^{\prime}, \\
& y^{\prime \prime}=-\lambda(\beta+1) y^{\beta-\alpha} x^{\prime}+\frac{\alpha}{y}\left(x^{\prime}\right)^{2} .
\end{aligned}
$$

Hence, using (2.65), we find for the curvature $\kappa(s)$ of the curve $(x(s), y(s)),(s \in(0, L))$,

$$
\begin{aligned}
\kappa & =\frac{-x^{\prime} y^{\prime \prime}+y^{\prime} x^{\prime \prime}}{\left(\left(x^{\prime}\right)^{2}+\left(y^{\prime}\right)^{2}\right)^{3 / 2}}=-x^{\prime} y^{\prime \prime}+y^{\prime} x^{\prime \prime} \\
& =-\frac{\alpha}{y} x^{\prime}+\lambda(\beta+1) y^{\beta-\alpha}=\lambda y^{\beta-\alpha}(\beta+1-\alpha)-\alpha \mathrm{d} y^{-1-\alpha} .
\end{aligned}
$$

The last expression is positive by (2.68) and (2.69), which means that $\Omega$ is strictly convex. The Lemma is proved.

Lemma 2.13. Assume (2.25) and (2.26), and let $\partial \Omega$ be given by (2.51)-(2.56). Then $y^{-}=0$.

Proof. Supposing that $y^{-}>0$, we will argue by contradiction. We proceed in 3 steps.

\section{Step 1: Another parametrization of $\partial \Omega$ :}

Let

$$
x_{0}:=\sup \{x:(x, y) \in \partial \Omega\} .
$$

Since $\Omega$ is strictly convex, there are functions $u_{1}, u_{2} \in C^{2}\left[0, x_{0}\right) \cap C\left[0, x_{0}\right]$ such that

$$
\begin{aligned}
& \partial \Omega \cap\{(x, y): x>0, y>0\}=\left\{(x, y): u_{1}(x)<y<u_{2}(x), 0<x<x_{0}\right\}, \\
& u_{1}(0)=y^{-}, u_{2}(0)=y^{+}, \\
& u_{1}\left(x_{0}\right)=u_{2}\left(x_{0}\right)=: y_{0}, \\
& u_{1}^{\prime}(0)=u_{2}^{\prime}(0)=0 .
\end{aligned}
$$

Furthermore, the Euler equations (2.52), (2.53) lead to

$$
\begin{aligned}
& \frac{\alpha u_{1}^{\alpha-1}}{\sqrt{1+\left(u_{1}^{\prime}\right)^{2}}}-\frac{u_{1}^{\alpha} u_{1}^{\prime \prime}}{\left(1+\left(u_{1}^{\prime}\right)^{2}\right)^{3 / 2}}=-\lambda(\beta+1) u_{1}^{\beta}, \\
& \frac{\alpha u_{2}^{\alpha-1}}{\sqrt{1+\left(u_{2}^{\prime}\right)^{2}}}-\frac{u_{2}^{\alpha} u_{2}^{\prime \prime}}{\left(1+\left(u_{2}^{\prime}\right)^{2}\right)^{3 / 2}}=\lambda(\beta+1) u_{2}^{\beta} .
\end{aligned}
$$

Using (2.73) and the fact that

$$
\begin{aligned}
& \lim _{x \rightarrow x_{0}} u_{1}^{\prime}(x)=+\infty \quad \text { and } \\
& \lim _{x \rightarrow x_{0}} u_{2}^{\prime}(x)=-\infty
\end{aligned}
$$


lead to

$$
\begin{aligned}
& \frac{u_{1}^{\alpha}}{\sqrt{1+\left(u_{1}^{\prime}\right)^{2}}}=\lambda\left(y_{0}^{\beta+1}-u_{1}^{\beta+1}\right), \\
& \frac{u_{2}^{\alpha}}{\sqrt{1+\left(u_{2}^{\prime}\right)^{2}}}=\lambda\left(u_{2}^{\beta+1}-y_{0}^{\beta+1}\right) .
\end{aligned}
$$

Finally, the boundary conditions (2.72)-(2.74) lead to the following formulas:

$$
\begin{aligned}
y_{0}^{\beta+1} & =\frac{\left(y^{-}\right)^{\beta+1}\left(y^{+}\right)^{\alpha}+\left(y^{+}\right)^{\beta+1}\left(y^{-}\right)^{\alpha}}{\left(y^{-}\right)^{\alpha}+\left(y^{+}\right)^{\alpha}}, \\
\lambda & =\frac{\left(y^{-}\right)^{\alpha}+\left(y^{+}\right)^{\alpha}}{\left(y^{+}\right)^{\beta+1}-\left(y^{-}\right)^{\beta+1}} .
\end{aligned}
$$

\section{Step 2: Curvature:}

In the following, we will refer to points $\left(x, u_{1}(x)\right)$ as points of the 'lower curve' and to points $\left(x, u_{2}(x)\right)$ as points of the 'upper curve', $\left(x \in\left(0, x_{0}\right)\right)$.

The signed curvature $\kappa$ (see (2.70)) can be expressed in terms of the functions $u_{1}$ and $u_{2}$. More precisely, we have

$$
\kappa=\left\{\begin{array}{ll}
\frac{u_{1}^{\prime \prime}}{\left(1+\left(u_{1}^{\prime}\right)^{2}\right)^{3 / 2}} & \text { on the lower curve } \\
-\frac{u_{2}^{\prime \prime}}{\left(1+\left(u_{2}^{\prime}\right)^{2}\right)^{3 / 2}} & \text { on the upper curve }
\end{array} .\right.
$$

Accordingly, we will write

$$
\begin{aligned}
\kappa_{1}(x) & :=\frac{u_{1}^{\prime \prime}}{\left(1+\left(u_{1}^{\prime}\right)^{2}\right)^{3 / 2}}, \\
\kappa_{2}(x) & :=-\frac{u_{2}^{\prime \prime}}{\left(1+\left(u_{2}^{\prime}\right)^{2}\right)^{3 / 2}}, \quad\left(x \in\left(0, x_{0}\right)\right) .
\end{aligned}
$$

Finally, let $s_{0} \in(0, L)$ be taken such that $y\left(s_{0}\right)=y_{0}$ and $x\left(s_{0}\right)=x_{0}$. Then formula (2.65) taken at $s=s_{0}$ leads to

$$
d=-\lambda y_{0}^{\beta+1}
$$

Plugging this into (2.70) we find

$$
\kappa(s)=\lambda\left((\beta+1-\alpha) y^{\beta-\alpha}+\alpha y^{-1-\alpha} y_{0}^{\beta+1}\right), \quad(s \in[0, L]) .
$$

Differentiating (2.85) we evaluate

$$
\kappa^{\prime}(s)=-\lambda y^{-2-\alpha} y^{\prime}\left(\alpha(\alpha+1) y_{0}^{\beta+1}-(\beta+1-\alpha)(\beta-\alpha) y^{\beta+1}\right) .
$$

Since $y^{\prime}(s)<0$ for $s \in(0, L)$, this in particular implies

$$
\kappa^{\prime}(s)>0 \quad \forall s \in[0, L], \quad \text { if } \beta \leq \alpha .
$$




\section{Step 4:}

We claim that

$$
\kappa_{1}(x)>\kappa_{2}(x) \quad \forall x \in\left(0, x_{0}\right) .
$$

First observe that (2.88) immediately follows from (2.87) if $\alpha \geq \beta$. Thus it remains to consider the case

$$
0<\alpha<\beta<2 \alpha .
$$

From $(2.86)$ and the fact that $y(s)<y_{0}$ for $s \in\left[s_{0}, L\right]$ we find that

$$
\begin{aligned}
\kappa^{\prime}(s) & \geq-\lambda y^{\prime} y^{-2-\alpha} y_{0}^{\beta+1}[\alpha(\alpha+1)-(\beta+1-\alpha)(\beta-\alpha)] \\
& =-\lambda y^{\prime} y^{-2-\alpha} y_{0}^{\beta+1}(2 \alpha-\beta)(\beta+1) \\
& >0 \quad \forall s \in\left[s_{0}, L\right) .
\end{aligned}
$$

This means that

$$
\kappa_{1}(x)>\kappa_{2}(x) \quad \forall x \in\left(x_{0}-\varepsilon, x_{0}\right)
$$

for some (small) $\varepsilon>0$. Now assume that (2.88) does not hold. By (2.91) there exists a number $x_{1} \in\left(0, x_{0}\right)$ such that

$$
\begin{aligned}
& \kappa_{1}(x)>\kappa_{2}(x) \quad \forall x \in\left(x_{1}, x_{0}\right), \quad \text { and } \\
& \kappa_{1}\left(x_{1}\right)=\kappa_{2}\left(x_{1}\right) .
\end{aligned}
$$

We claim that (2.92) implies that

$$
u_{1}^{\prime}\left(x_{1}\right)<-u_{2}^{\prime}\left(x_{1}\right)
$$

To prove (2.94), observe first that

$$
\lim _{x \rightarrow x_{0}} u_{1}^{\prime}(x)=\lim _{x \rightarrow x_{0}}\left(-u_{2}^{\prime}(x)\right)=+\infty .
$$

Then, integrating $(2.92)$ over $\left(x_{1}, x_{0}\right)$ leads to

$$
\begin{aligned}
& 1-\frac{u_{1}^{\prime}\left(x_{1}\right)}{\sqrt{1+\left(u_{1}^{\prime}\left(x_{1}\right)\right)^{2}}}=\int_{x_{1}}^{x_{0}}\left(\frac{u_{1}^{\prime}(x)}{\sqrt{1+\left(u_{1}^{\prime}(x)\right)^{2}}}\right)^{\prime} \mathrm{d} x \\
= & \int_{x_{1}}^{x_{0}} \frac{u_{1}^{\prime \prime}(x)}{\left[1+\left(u_{1}^{\prime}(x)\right)^{2}\right]^{3 / 2}} \mathrm{~d} x=\int_{x_{1}}^{x_{0}} \kappa_{1}(x) \mathrm{d} x \\
> & \int_{x_{1}}^{x_{0}} \kappa_{2}(x) \mathrm{d} x=\int_{x_{1}}^{x_{0}} \frac{-u_{2}^{\prime \prime}(x)}{\left[1+\left(u_{2}^{\prime}(x)\right)^{2}\right]^{3 / 2}} \mathrm{~d} x \\
= & \int_{x_{1}}^{x_{0}}\left(\frac{-u_{2}^{\prime}(x)}{\sqrt{1+\left(u_{2}^{\prime}(x)\right)^{2}}}\right)^{\prime} \mathrm{d} x=1+\frac{u_{2}^{\prime}\left(x_{1}\right)}{\sqrt{1+\left(u_{2}^{\prime}\left(x_{1}\right)\right)^{2}}},
\end{aligned}
$$


which implies (2.94). Now, from (2.94) we deduce

$$
\frac{1}{\sqrt{1+\left(u_{1}^{\prime}\left(x_{1}\right)\right)^{2}}}>\frac{1}{\sqrt{1+\left(u_{2}^{\prime}\left(x_{1}\right)\right)^{2}}} .
$$

Together with (2.79) and (2.80) we obtain from this

$$
\frac{y_{0}^{\beta+1}-\left(u_{1}\left(x_{1}\right)\right)^{\beta+1}}{\left(u_{1}\left(x_{1}\right)\right)^{\alpha}}>\frac{\left(u_{2}\left(x_{1}\right)\right)^{\beta+1}-y_{0}^{\beta+1}}{\left(u_{2}\left(x_{1}\right)\right)^{\alpha}},
$$

or, equivalently

$$
\left(u_{1}\left(x_{1}\right)\right)^{\beta+1-\alpha}+\left(u_{2}\left(x_{1}\right)\right)^{\beta+1-\alpha}<y_{0}^{\beta+1}\left(\left(u_{1}\left(x_{1}\right)\right)^{-\alpha}+\left(u_{2}\left(x_{1}\right)\right)^{-\alpha}\right) .
$$

Furthermore, multiplying $(2.75)$ by $\left(u_{1}\right)^{-\alpha}$, respectively $(2.76)$ by $\left(u_{2}\right)^{-\alpha}$, adding both equations and taking into account (2.93) leads to

$$
\frac{\alpha}{u_{1} \sqrt{1+\left(u_{1}^{\prime}\right)^{2}}}+\frac{\alpha}{u_{2} \sqrt{1+\left(u_{2}^{\prime}\right)^{2}}}=\lambda(\beta+1)\left(\left(u_{2}\right)^{\beta-\alpha}-\left(u_{1}\right)^{\beta-\alpha}\right) \quad \text { at } x=x_{1} .
$$

Using once more (2.79) and (2.80) then gives

$$
\frac{\alpha\left(y_{0}^{\beta+1}-\left(u_{1}\right)^{\beta+1}\right)}{\left(u_{1}\right)^{\alpha+1}}+\frac{\alpha\left(\left(u_{2}\right)^{\beta+1}-y_{0}^{\beta+1}\right)}{\left(u_{2}\right)^{\alpha+1}}=(\beta+1)\left(\left(u_{2}\right)^{\beta-\alpha}-\left(u_{1}\right)^{\beta-\alpha}\right),
$$

or equivalently,

$$
\alpha y_{0}^{\beta+1}\left(\left(u_{1}\right)^{-\alpha-1}-\left(u_{2}\right)^{-\alpha-1}\right)=(\beta+1-\alpha)\left(\left(u_{2}\right)^{\beta-\alpha}-\left(u_{1}\right)^{\beta-\alpha}\right) \quad \text { at } x=x_{1} .
$$

From this and (2.95) we then obtain

$$
\begin{aligned}
& (\beta+1-\alpha)\left(\left(u_{2}\right)^{\beta-\alpha}-\left(u_{1}\right)^{\beta-\alpha}\right) \\
> & \alpha\left(\left(u_{1}\right)^{-\alpha-1}-\left(u_{2}\right)^{-\alpha-1}\right) \frac{\left(u_{2}\right)^{\beta+1-\alpha}+\left(u_{1}\right)^{\beta+1-\alpha}}{\left(u_{1}\right)^{-\alpha}+\left(u_{2}\right)^{-\alpha}} \text { at } x=x_{1} .
\end{aligned}
$$

Setting

$$
z:=\frac{u_{1}\left(x_{1}\right)}{u_{2}\left(x_{1}\right)} \in(0,1)
$$

this leads to

$$
(\beta+1-\alpha)\left(z-z^{\beta+1-\alpha}+z^{\alpha+1}-z^{\beta+1}\right)>\alpha\left(1-z^{\alpha+1}+z^{\beta+1-\alpha}-z^{\beta+2}\right) .
$$

But this contradicts Lemma A (Appendix). This finishes the proof of (2.88).

Step 5: Since $\lim _{x \rightarrow x_{0}} u_{1}^{\prime}(x)=-\lim _{x \rightarrow x_{0}} u_{2}^{\prime}(x)=+\infty$, (2.88) implies that

$$
u_{1}^{\prime}(0)<-u_{2}^{\prime}(0)=0,
$$


which contradicts the boundary conditions (2.74). Hence we must have that $y^{-}=0$.

Now we are in a position to give a

Proof of Theorem 1.1: We split into two cases.

Case (i): Assume that

$$
\beta<2 \alpha .
$$

By Lemma 2.13 we have $y^{-}=0$. Now, since $x^{\prime}(L)=0$, equation (2.53) at $s=L$ yields

$$
d=0
$$

so that

$$
x^{\prime}=\lambda y^{\beta+1-\alpha} .
$$

In view of Remark 2.2 we may rescale $\Omega$ in such a way that $y^{+}=1$. Then (2.100) at $s=0$ gives $\lambda=1$. Since $y^{\prime}(s)<0$ for $s \in(0, L)$, we have $s=g(y)$ with a decreasing function $g \in C^{1}(0,1)$. Writing

$$
f(y):=x(g(y))
$$

we obtain

$$
-\frac{f^{\prime}(y)}{\sqrt{1+\left(f^{\prime}(y)\right)^{2}}}=y^{\beta+1-\alpha},
$$

and integrating this leads to (1.3) and (1.4).

Case (ii) Now assume that

$$
\beta=2 \alpha
$$

Since the case $\alpha=0$ is trivial, we may assume $\alpha>0$. Let us fix such $\alpha$.

First observe that for every smooth domain $U \subset \mathbb{R}_{+}^{2}$, the mapping

$$
\beta \longmapsto \mathcal{R}_{\alpha, \beta}(U), \quad(-1<\beta \leq 2 \alpha),
$$

is continuous. Furthermore, from Case (i) we see that the mapping

$$
\beta \longmapsto \mu(\alpha, \beta), \quad(-1<\beta<2 \alpha),
$$

is continuous, and the limit

$$
Z:=\lim _{\beta \rightarrow 2 \alpha-} \mu(\alpha, \beta)
$$

exists.

Now let $\Omega^{\star}$ be the domain that is given by formulas (1.3), (1.4), with $\beta=2 \alpha$. Then we also have

$$
Z=\mathcal{R}_{\alpha, 2 \alpha}\left(\Omega^{\star}\right)
$$


which implies that $Z \geq \mu(\alpha, 2 \alpha)$.

Assume that

$$
Z>\mu(\alpha, 2 \alpha) .
$$

Then there is a smooth set $\Omega^{\prime} \subset \mathbb{R}_{+}^{2}$ such that also

$$
Z>\mathcal{R}_{\alpha, 2 \alpha}\left(\Omega^{\prime}\right)
$$

But by (2.103) this implies that

$$
\mathcal{R}_{\alpha, \beta}\left(\Omega^{\prime}\right)<\mu(\alpha, \beta),
$$

when $\beta<2 \alpha$ and $|\beta-2 \alpha|$ is small, which is impossible. Hence we have that

$$
Z=\mu(\alpha, 2 \alpha)=\mathcal{R}_{\alpha, 2 \alpha}\left(\Omega^{\star}\right) .
$$

This finishes the proof of the Theorem.

Finally we evaluate $\mu(\alpha, \beta)$. Put $\gamma:=\beta+1-\alpha(>0)$. With the Beta function $B$ and the function $f$ given by (1.4) we have

$$
\begin{aligned}
P_{\alpha}\left(\Omega^{\star}\right) & =2 \int_{0}^{1} y^{\alpha} \sqrt{1+\left(f^{\prime}(y)\right)^{2}} \mathrm{~d} y=2 \int_{0}^{1} \frac{y^{\alpha}}{\sqrt{1-y^{2 \gamma}}} \mathrm{d} y \\
& =\frac{1}{\gamma} \int_{0}^{1} \frac{z^{\frac{\alpha+1}{2 \gamma}-1} d z}{\sqrt{1-z}}=\frac{1}{\gamma} \cdot B\left(\frac{\alpha+1}{2 \gamma}, \frac{1}{2}\right),
\end{aligned}
$$

and

$$
\begin{aligned}
A_{\beta}\left(\Omega^{\star}\right) & =2 \int_{0}^{1} y^{\beta} f(y) \mathrm{d} y=-\frac{2}{\beta+1} \int_{0}^{1} y^{\beta+1} f^{\prime}(y) \mathrm{d} y \\
& =\frac{2}{\beta+1} \int_{0}^{1} \frac{y^{2 \gamma}}{\sqrt{1-y^{2 \gamma}}} \mathrm{d} y=\frac{1}{\gamma(\beta+1)} \int_{0}^{1} \frac{z^{\frac{\alpha+1}{2 \gamma}}}{\sqrt{1-z}} d z \\
& =\frac{1}{\gamma(\beta+1)} B\left(1+\frac{\alpha+1}{2 \gamma}, \frac{1}{2}\right) .
\end{aligned}
$$

Using the identity

$$
B(a+1, b)=B(a, b) \cdot \frac{a}{a+b}, \quad(\operatorname{Re}(a)>0, \operatorname{Re}(b)>0)
$$

we obtain

$$
\begin{aligned}
\mu(\alpha, \beta) & =\mathcal{R}_{\alpha, \beta}\left(\Omega^{\star}\right)=\frac{P_{\alpha}\left(\Omega^{\star}\right)}{\left[A_{\beta}\left(\Omega^{\star}\right)\right]^{\frac{\alpha+1}{\beta+2}}} \\
& =\frac{1}{\gamma} \cdot B\left(\frac{\alpha+1}{2 \gamma}, \frac{1}{2}\right) \cdot\left[\frac{1}{\gamma(\beta)} \cdot B\left(1+\frac{\alpha+1}{2 \gamma}, \frac{1}{2}\right)\right]^{-\frac{\alpha+1}{\beta+2}} \\
& =\gamma^{\frac{\alpha+1}{\beta+2}-1} \cdot\left[\frac{(\beta+1)(\beta+2)}{\alpha+1}\right]^{\frac{\alpha+1}{\beta+2}} \cdot\left[B\left(\frac{\alpha+1}{2 \gamma}, \frac{1}{2}\right)\right]^{\frac{\gamma}{\beta+2}}
\end{aligned}
$$


which is (1.5). In case of $\beta=2 \alpha$ this leads to

$$
\mu(\alpha, 2 \alpha)=(\alpha+1)^{-\frac{1}{2}} \cdot[2(2 \alpha+1)]^{\frac{1}{2}} \cdot\left[B\left(\frac{1}{2}, \frac{1}{2}\right)\right]^{\frac{1}{2}}=\sqrt{\frac{2 \pi(2 \alpha+1)}{\alpha+1}} .
$$

Remark 2.14. It is also well-known that the isoperimetric inequality is equivalent to the following functional inequality, (see [1], Lemma 3.5).

$$
\iint_{\mathbb{R}_{+}^{2}}|\nabla u| y^{\alpha} \mathrm{d} x \mathrm{~d} y \geq \mu(\alpha, \beta)\left(\iint_{\mathbb{R}_{+}^{2}}|u|^{\frac{\beta+2}{\alpha+1}} y^{\beta} \mathrm{d} x \mathrm{~d} y\right)^{\frac{\alpha+1}{\beta+2}}, \quad \forall u \in C_{0}^{\infty}\left(\mathbb{R}^{2}\right) .
$$

Remark 2.15. In [40] (see also [28-30]) the authors obtain a sharp isoperimetric inequality in the Grushin plane, and they also compute the corresponding isoperimetric sets. Their proof consists of three steps. First, performing a certain transformation in one of the coordinates, the problem becomes equivalent to the task of minimizing the (unweighted) Euclidean perimeter of sets $U \subset \mathbb{R}^{2}$ having fixed weighted measure $\iint_{U}|y|^{\beta} \mathrm{d} x \mathrm{~d} y$, where $\beta \in(-1,0]$. Then, using two consecutive Steiner symmetrizations in the $x$ - and the $y$-variable, the perimeter of an admissible set $U$ decreases while the weighted measure increases. This means that the problem

can be further reduced to sets which are Steiner symmetric in both variables. In turn, the boundary of the minimizer must be a polar graph which is then found in the last step of the proof.

In view of the symmetry of the minimizer w.r.t. the $x$-axis, this isoperimetric result can also be read off from Theorem 1.1 when $\alpha=0$ and $\beta \in(-1,0]$. Note however, that in the study of our problem $(\mathbf{P})$ extra difficulties appeared because rearrangement arguments in the $y$-variable failed in the general case.

\section{Applichtions}

In this section we firstly show that our isoperimetric inequality implies a sharp estimate of the so-called weighted Cheeger constant.

Then we deduce an estimate of the first eigenvalue to a degenerate elliptic Dirichlet boundary values problem. We begin by introducing some function spaces that will be used in the sequel.

Let $\Omega$ be an open subset of $\mathbb{R}_{+}^{2}$ and $p \in[1,+\infty)$.

By $L^{p}\left(\Omega ; y^{\beta}\right)$ we denote the weighted Hölder space of measurable functions $u: \Omega \rightarrow \mathbb{R}$ such that

$$
\|u\|_{L^{p}\left(\Omega ; y^{\beta}\right)}:=\left(\iint_{\Omega}|u|^{p} y^{\beta} \mathrm{d} x \mathrm{~d} y\right)^{1 / p}<+\infty .
$$

Then let $W^{1, p}\left(\Omega ; y^{\alpha}, y^{\beta}\right)$ be the weighted Sobolev space of all functions $u \in L^{p}\left(\Omega ; y^{\beta}\right)$ possessing weak first partial derivatives which belong to $L^{p}\left(\Omega ; y^{\beta}\right)$. A norm in $W^{1, p}\left(\Omega ; y^{\alpha}, y^{\beta}\right)$ is given by

$$
\|u\|_{W^{1, p}\left(\Omega ; y^{\alpha}, y^{\beta}\right)}:=\||\nabla u|\|_{L^{p}\left(\Omega ; y^{\alpha}\right)}+\|u\|_{L^{p}\left(\Omega ; y^{\beta}\right)} .
$$

For any function $u \in L^{1}\left(\Omega ; y^{\beta}\right)$ we write

$$
|D u|\left(\Omega ; y^{\alpha}\right):=\sup \left\{\iint_{\Omega} u \operatorname{div}\left(\mathbf{v} y^{\alpha}\right) \mathrm{d} x \mathrm{~d} y: \mathbf{v} \in C_{0}^{\infty}\left(\Omega, \mathbb{R}^{2}\right),|\mathbf{v}| \leq 1\right\} .
$$

Then let $B V\left(\Omega ; y^{\alpha}, y^{\beta}\right)$ be the weighted BV-space of all functions $u \in L^{1}\left(\Omega ; y^{\beta}\right)$ such that $|D u|\left(\Omega ; y^{\alpha}\right)<+\infty$. A norm on $B V\left(\Omega ; y^{\alpha}, y^{\beta}\right)$ is given by

$$
\|u\|_{B V\left(\Omega ; y^{\alpha}, y^{\beta}\right)}:=|D u|\left(\Omega ; y^{\alpha}\right)+\|u\|_{L^{1}\left(\Omega ; y^{\beta}\right)} .
$$


Let us explicitly remark that for an open bounded set $\Omega \subset \mathbb{R}_{+}^{2}$ the following equality holds

$$
P_{\alpha}(\Omega)=\left|D \chi_{\Omega}\right|\left(\mathbb{R}_{+}^{2} ; y^{\alpha}\right)
$$

Finally let $X$ be the set of all the functions $w \in C^{1}(\bar{\Omega})$ that vanish in a neighborhood of $\partial \Omega \cap \mathbb{R}_{+}^{2}$. Then $V^{p}\left(\Omega ; y^{\alpha}, y^{\beta}\right)$ will denote the closure of $X$ in the norm of $W^{1, p}\left(\Omega ; y^{\alpha}, y^{\beta}\right)$.

Finally we denote by $\Omega^{\star}$ the set $t \Omega^{\star}$, for $t>0$, such that $A_{\beta}(\Omega)=A_{\beta}\left(\Omega^{\star}\right)$.

\subsection{Weighted Cheeger sets}

We define the weighted Cheeger constant of an open bounded set $\Omega \subset \mathbb{R}_{+}^{2}$ as

$$
h_{\alpha, \beta}(\Omega)=\inf \left\{\frac{P_{\alpha}(E)}{A_{\beta}(E)}: E \subset \Omega, 0<A_{\beta}(E)<+\infty\right\} .
$$

(see also $[34,47])$

We firstly prove that the existence of an ammissible set which realizes the minimum in (3.1) (see also [47]).

Lemma 3.1. Assume $0 \leq \alpha<\beta+1$ and $\beta \leq 2 \alpha$. For any open bounded set $\Omega \subset \subset \mathbb{R}_{+}^{2}$, there exists at least one set $M \subseteq \Omega$, the so-called weighted Cheeger set, such that

$$
h_{\alpha, \beta}(\Omega)=\frac{P_{\alpha}(M)}{A_{\beta}(M)} .
$$

Proof. Since $\Omega$ is open, $h_{\alpha, \beta}(\Omega)$ is finite: Indeed, it is easy to verify that for any ball $B$ with $B \subset \subset \Omega$, the ratio $\frac{P_{\alpha}(B)}{A_{\beta}(B)}$ is finite.

Let $\left\{E_{k}\right\}$ be a minimizing sequence for (3.1). Since $\Omega$ is bounded, we have

$$
A_{\beta}\left(E_{k}\right)=\iint_{E_{k}} y^{\beta} \mathrm{d} x \mathrm{~d} y \leq A_{\beta}(\Omega)=\iint_{\Omega} y^{\beta} \mathrm{d} x \mathrm{~d} y<+\infty .
$$

Now fix $\varepsilon>0$. There exists an index $\bar{k}$ such that

$$
\left|h_{\alpha, \beta}(\Omega)-\frac{P_{\alpha}\left(E_{k}\right)}{A_{\beta}\left(E_{k}\right)}\right|<\varepsilon, \quad \forall k>\bar{k}
$$

Since $\Omega$ is bounded, for all $k>\bar{k}$, we get

$$
P_{\alpha}\left(E_{k}\right)<\left(\varepsilon+h_{\alpha, \beta}(\Omega)\right) A_{\beta}\left(E_{k}\right) \leq\left(\varepsilon+h_{\alpha, \beta}(\Omega)\right) A_{\beta}(\Omega) \equiv C
$$

This implies

$$
\left|D \chi_{E_{k}}\left(\mathbb{R}_{+}^{2}, y^{\alpha}\right)\right|=P_{\alpha}\left(E_{k}\right) \leq C \quad \forall k>\bar{k}
$$

Hence $\left\{E_{k}\right\}$ is an equibounded family in weighted $B V\left(\Omega ; y^{\alpha}, y^{\beta}\right)$-norm. Thus by Lemma B (Appendix), up to subsequences, $\left\{\chi\left(E_{k}\right)\right\}$ converges in the weighted $L^{1}\left(\Omega ; y^{\beta}\right)$-norm and pointwise a.e. to a function $u$. Moreover there exists a subset $M \subseteq \Omega$ such that $u=\chi_{M}$. 
Since $\left\{E_{k}\right\}$ is a minimizing sequence, by lower semicontinuity of perimeter $P_{\alpha}$ and Lebesgue dominated convergence theorem, we get

$$
h_{\alpha, \beta}(\Omega)=\lim _{k \rightarrow+\infty} \frac{P_{\alpha}\left(E_{k}\right)}{A_{\beta}\left(E_{k}\right)} \geq \frac{P_{\alpha}(M)}{A_{\beta}(M)} .
$$

It remains to prove that $M$ is an admissible set, that is we need to prove that

$$
A_{\beta}(M)>0
$$

Assume by contradiction that $A_{\beta}(M)=0$. This implies that $\lim _{k \rightarrow+\infty} A_{\beta}\left(E_{k}\right)=0$.

Now for a fixed $\eta>0$ consider the set

$$
E_{k, \eta}=\left\{(x, y) \in E_{k}: y>\eta\right\}
$$

Now the following inequality holds true

$$
A_{\beta}\left(E_{k, \eta}\right)<\delta^{\beta} A_{0}\left(E_{k, \eta}\right), \quad P_{\alpha}\left(E_{k, \eta}\right)<\eta^{\alpha} P_{0}\left(E_{k, \eta}\right)
$$

where $\delta=\eta$, if $\beta \leq 0$ and $\delta=R$ for a suitable $R>0$ such that a ball $B_{R}$ of radius $R$ contains $\Omega$, if $\beta>0$. Denote by $B_{r_{k}, \eta}$ a ball of radius $r_{k}$ having the same Lebesgue measure $A_{0}\left(E_{k, \eta}\right)$ of $E_{k, \eta}$. By the classical isoperimetric inequality, we get

$$
\frac{P_{\alpha}\left(E_{k, \eta}\right)}{A_{\beta}\left(E_{k, \eta}\right)} \geq \frac{\eta^{\alpha} P_{\alpha}\left(E_{k, \eta}\right)}{\delta^{\beta} A_{0}\left(B_{r_{k}, \eta}\right)}=\frac{\eta^{\alpha} P_{\alpha}\left(B_{r_{k}, \eta}\right)}{\delta^{\beta} A_{0}\left(B_{r_{k}, \eta}\right)}=\frac{2 \eta^{\alpha}}{\delta^{\beta} r_{k}} \rightarrow+\infty, \quad \text { as } k \text { goes to }+\infty .
$$

This yields a contradiction. Therefore (3.4) holds true and the conclusion follows.

We recall that $\Omega^{\star}$ is the set $t \Omega^{\star}$, for $t>0$, such that $A_{\beta}(\Omega)=A_{\beta}\left(\Omega^{\star}\right)$.

Once we have proved the existence of a weighted Cheeger set, we can obtain the following result.

Theorem 3.2. Assume $0 \leq \alpha<\beta+1$ and $\beta \leq 2 \alpha$ and $\alpha<\beta+1$, and let $\Omega$ be a bounded open subset $\mathbb{R}_{+}^{2}$. Then the following estimate holds true

$$
h_{\alpha, \beta}(\Omega) \geq h_{\alpha, \beta}\left(\Omega^{\star}\right)=\frac{P_{\alpha}\left(\Omega^{\star}\right)}{A_{\beta}\left(\Omega^{\star}\right)} .
$$

Proof. Let $E$ be a nonempty subset of $\Omega$ with $P_{\alpha}(E)<+\infty$. By our isoperimetric inequality Theorem 1.1 and since $E^{\star} \subset \Omega^{\star}$ with $A_{\beta}(E)=A_{\beta}\left(E^{\star}\right)$, we have that

$$
\frac{P_{\alpha}(E)}{A_{\beta}(E)} \geq \frac{P_{\alpha}\left(E^{\star}\right)}{A_{\beta}\left(E^{\star}\right)} \geq h_{\alpha, \beta}\left(\Omega^{\star}\right) .
$$

It remains to prove the equality in (3.5). Let $F$ be a nonempty subset of $\Omega^{\star}$. Then we have $F^{\star} \subset \Omega^{\star}$ and

$$
\frac{P_{\alpha}(F)}{A_{\beta}(F)} \geq \frac{P_{\alpha}\left(F^{\star}\right)}{A_{\beta}\left(F^{\star}\right)}=t^{\beta+1-\alpha} \frac{P_{\alpha}\left(t F^{\star}\right)}{A_{\beta}\left(t F^{\star}\right)}
$$


for all $t>0$. Since $F^{\star} \subset \Omega^{\star}$, there exists $t \geq 1$ such that $t F^{\star}=\Omega^{\star}$. Therefore

$$
\frac{P_{\alpha}(F)}{A_{\beta}(F)} \geq t^{\beta+1-\alpha} \frac{P_{\alpha}\left(\Omega^{\star}\right)}{A_{\beta}\left(\Omega^{\star}\right)}
$$

which proves the equality in (3.5).

Remark 3.3. Theorem 3.1 could be stated as an estimate of the first eigenvalue of the weighted 1-laplacian.

\subsection{A nonlinear eigenvalue problem}

Let $\Omega \subset \mathbb{R}_{+}^{2}$ be a bounded domain and let $p \in(1,+\infty)$. We consider the following weighted eigenvalue problem

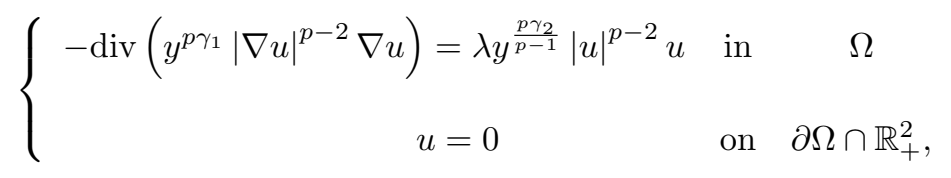

where

$$
\gamma_{1}=\alpha-\frac{p-1}{p} \beta \text { and } \gamma_{2}=\frac{p-1}{p} \beta
$$

By a solution to problem (3.8) we mean a function $u \in V^{p}\left(\Omega ; y^{p \gamma_{1}}, y^{\frac{p \gamma_{2}}{p-1}}\right)$ such that

$$
\iint_{\Omega}|\nabla u|^{p-2} \nabla u \nabla \psi y^{p \gamma_{1}} \mathrm{~d} x \mathrm{~d} y=\lambda \iint_{\Omega}|u|^{p-2} u \psi y^{\frac{p \gamma_{2}}{p-1}} \mathrm{~d} x \mathrm{~d} y
$$

for all function $\psi \in C^{1}(\bar{\Omega})$ such that $\psi=0$ on $\partial \Omega \cap \mathbb{R}_{+}^{2}$.

Let us denote by $T$ the range of values of $\alpha$ and $\beta$ for which the isoperimetric inequality holds true. We have that

$$
(\alpha, \beta) \in T=\{\alpha \geq 0\} \cap\{\beta>\alpha-1\} \cap\{\beta \leq 2 \alpha\}
$$

if and only if

$$
\left(\gamma_{1}, \gamma_{2}\right) \in U:=\left\{\gamma_{1}+\gamma_{2} \geq 0\right\} \cap\left\{\frac{p \gamma_{2}}{p-1}>\gamma_{1}+\gamma_{2}-1\right\} \cap\left\{\frac{p \gamma_{2}}{p-1} \leq 2\left(\gamma_{1}+\gamma_{2}\right)\right\} .
$$

Furthermore the smallest eigenvalue of problem $(3.8), \lambda_{1, p}^{\gamma_{1}, \gamma_{2}}(\Omega)$, has the following variational characterization

$$
\lambda_{1, p}^{\gamma_{1}, \gamma_{2}}(\Omega)=\min \left\{\frac{\iint_{\Omega}|\nabla u|^{p} y^{p \gamma_{1}} \mathrm{~d} x \mathrm{~d} y}{\iint_{\Omega} u^{p} y^{\frac{p \gamma_{2}}{p-1}} \mathrm{~d} x \mathrm{~d} y} \text { with } u \in V^{p}\left(\Omega ; y^{p \gamma_{1}}, y^{\frac{p \gamma_{2}}{p-1}}\right) \backslash\{0\}\right\} .
$$


Indeed, see e.g. Theorem 8.9 in [31], for any $\left(\gamma_{1}, \gamma_{2}\right) \in U$, the following compact embedding holds true

$$
V^{p}\left(\Omega ; y^{p \gamma_{1}}, y^{\frac{p \gamma_{2}}{p-1}}\right) \hookrightarrow \hookrightarrow L^{p}\left(\Omega ; y^{\frac{p \gamma_{2}}{p-1}}\right)
$$

By adapting the arguments used in [22, 35], we obtain the following result

Theorem 3.4. Let $\left(\gamma_{1}, \gamma_{2}\right) \in U$, then the following estimate holds true

$$
\lambda_{1, p}^{\gamma_{1}, \gamma_{2}}(\Omega) \geq \frac{1}{p^{p}}\left[h_{\alpha, \beta}\left(\Omega^{\star}\right)\right]^{p}=\frac{1}{p^{p}}\left[\frac{P_{\alpha}\left(\Omega^{\star}\right)}{A_{\beta}\left(\Omega^{\star}\right)}\right]^{p} .
$$

Remark 3.5. If $\alpha=\beta>0$ and $p=2$, a Faber-Krahn type inequality for $\lambda_{1, p}^{\gamma_{1}, \gamma_{2}}(\Omega)$ holds true (see [37]). Indeed in this case, we have $\alpha=2 \gamma_{1}=2 \gamma_{2}=\beta$.

Proof of Theorem 3.4 We claim that

$$
\lambda_{1, p}^{\gamma_{1}, \gamma_{2}}(\Omega) \geq \frac{\left[h_{\alpha, \beta}(\Omega)\right]^{p}}{p^{p}}
$$

where $h_{\alpha, \beta}(\Omega)$ is defined in (3.1). Let $u$ be an eigenfunction corresponding to $\lambda_{1, p}^{\gamma_{1}, \gamma_{2}}(\Omega)$. Hölder's inequality gives

$$
\begin{aligned}
\iint_{\Omega}|\nabla u| u^{p-1} y^{\alpha} \mathrm{d} x \mathrm{~d} y & =\iint_{\Omega}|\nabla u| u^{p-1} y^{\gamma_{1}} y^{\gamma_{2}} \mathrm{~d} x \mathrm{~d} y \\
& \leq\left(\iint_{\Omega}|\nabla u|^{p} y^{p \gamma_{1}} \mathrm{~d} x \mathrm{~d} y\right)^{\frac{1}{p}}\left(\iint_{\Omega} u^{p} y^{\frac{\gamma_{2} p}{p-1}} \mathrm{~d} x \mathrm{~d} y\right)^{\frac{p-1}{p}},
\end{aligned}
$$

and therefore

$$
\iint_{\Omega}|\nabla u|^{p} y^{p \gamma_{1}} \mathrm{~d} x \geq \frac{\left(\iint_{\Omega}|\nabla u| u^{p-1} y^{\alpha} \mathrm{d} x \mathrm{~d} y\right)^{p}}{\left(\iint_{\Omega} u^{p} y^{\beta} \mathrm{d} x \mathrm{~d} y\right)^{p-1}}=\frac{\left(\iint_{\Omega}\left|\nabla\left(u^{p}\right)\right| y^{\alpha} \mathrm{d} x \mathrm{~d} y\right)^{p}}{p^{p}\left(\iint_{\Omega} u^{p} y^{\beta} \mathrm{d} x \mathrm{~d} y\right)^{p-1}} .
$$

Let $f:=u^{p}$, the previous inequality gives

$$
\frac{\iint_{\Omega}|\nabla u|^{p} y^{p \gamma_{1}} \mathrm{~d} x \mathrm{~d} y}{\iint_{\Omega} u^{p} y^{\beta} \mathrm{d} x \mathrm{~d} y} \geq \frac{\left(\iint_{\Omega}|\nabla f| y^{\alpha} \mathrm{d} x \mathrm{~d} y\right)^{p}}{p^{p}\left(\iint_{\Omega} f y^{\beta} \mathrm{d} x \mathrm{~d} y\right)^{p}} .
$$

On the othe hand Coarea formula yields 


$$
\begin{aligned}
& \frac{\left(\iint_{\Omega}|\nabla f| y^{\alpha} \mathrm{d} x \mathrm{~d} y\right)^{p}}{p^{p}\left(\iint_{\Omega} f y^{\beta} \mathrm{d} x \mathrm{~d} y\right)^{p}}=\frac{1}{p^{p}\left(\iint f y^{\beta} \mathrm{d} x \mathrm{~d} y\right)^{p}}\left(\int_{0}^{\max f} P_{\alpha}(\{f(x)>t\}) \mathrm{d} t\right)^{p} \\
& \geq \frac{p^{p}\left(\iint_{\Omega} f y^{\beta} \mathrm{d} x \mathrm{~d} y\right)^{p}}{\left(\int_{0}^{\max f} \frac{P_{\alpha}(\{f(x)>t\}) \mathrm{d} t}{A_{\beta}(\{f(x)>t\})} A_{\beta}(\{f(x)>t\}) \mathrm{d} t\right)^{p}} \\
& \geq \frac{p^{p}\left(\iint_{\Omega} f y^{\beta} \mathrm{d} x \mathrm{~d} y\right)^{p}}{\left(\int_{0}^{\max f} h_{\alpha, \beta}(\{f(x)>t\}) A_{\beta}(\{f(x)>t\}) \mathrm{d} t\right)^{p}} \\
& \quad \geq \frac{\left[h_{\alpha, \beta}(\Omega)\right]^{p}}{\left(\iint_{\Omega} f y^{\beta} \mathrm{d} x \mathrm{~d} y\right)^{p}}\left(\int_{0}^{\max f} A_{\beta}(\{f(x)>t\}) \mathrm{d} t\right)^{p}=\frac{\left[h_{\alpha, \beta}(\Omega)\right]^{p}}{p^{p}} .
\end{aligned}
$$

The claim is hence proved. It immediately implies Theorem 3.4, thanks to (3.5).

\section{Appendix A.}

We prove two technical results.

Lemma A: Let $\alpha, \beta \in \mathbb{R}$ with $0<\alpha \leq \beta \leq 2 \alpha$. Then

$$
\begin{aligned}
& \alpha\left(1-z^{\alpha+1}+z^{\beta+1-\alpha}-z^{\beta+2}\right)+(\beta+1-\alpha)\left(-z+z^{\beta+1-\alpha}-z^{\alpha+1}+z^{\beta+1}\right) \\
& \quad>0 \quad \forall z \in(0,1) .
\end{aligned}
$$

Proof. We fix $\alpha>0$ and define

$$
g(z, \beta):=\alpha\left(1-z^{\alpha+1}+z^{\beta+1-\alpha}-z^{\beta+2}\right)+(\beta+1-\alpha)\left(-z+z^{\beta+1-\alpha}-z^{\alpha+1}+z^{\beta+1}\right),
$$

and

$$
\begin{aligned}
h(z):= & g(z, 2 \alpha)=\alpha\left(1-z^{2 \alpha+2}\right)+(\alpha+1)\left(-z+z^{2 \alpha+1}\right), \\
& (z \in[0,1], \beta \in[\alpha, 2 \alpha]) .
\end{aligned}
$$


Then

$$
\begin{aligned}
& g(z, \alpha)=\alpha\left(1-z^{\alpha+1}\right)(1+z)>0 \\
& h(0)=\alpha, h(0)=0 \\
& h^{\prime}(z)=-2 \alpha(\alpha+1) z^{2 \alpha+1}+(\alpha+1)\left[-1+(2 \alpha+1) z^{2 \alpha}\right] \quad \text { and } \\
& h^{\prime \prime}(z)=2 \alpha(\alpha+1)(2 \alpha+1) z^{2 \alpha+1}(1-z)>0 .
\end{aligned}
$$

Hence $h^{\prime}(1)=0$ which together with (A.3) and (A.4) implies that

$$
h(z)=g(z, \alpha)>0 \quad \forall z \in(0,1)
$$

Furthermore we have

$$
\begin{aligned}
\frac{\partial g}{\partial \beta}(z, \beta)= & z^{\beta+1-\alpha}\left[\alpha\left(1-z^{1+\alpha}\right)+(\beta+1-\alpha)\left(1+z^{\alpha}\right)\right] \ln z \\
& -\left(1-z^{\beta}\right)\left(z+z^{\alpha+1}\right) \\
< & 0 \quad \text { if } z \in(0,1) \text { and } \beta \in[\alpha, 2 \beta] .
\end{aligned}
$$

Together with (A.2) and (A.4) this implies

$$
g(z, \alpha)>0 \quad \text { if } z \in(0,1) \text { and } \beta \in[\alpha, 2 \alpha]
$$

which is (A.1).

Lemma B: Let $\left\{u_{n}\right\} \subset B V\left(\Omega ; y^{\alpha}, y^{\beta}\right)$ be a bounded sequence. Then there exists a subsequence that converges in $L^{1}\left(\Omega ; y^{\beta}\right)$ and a.e. in $\Omega$ to some function $u$.

Proof. Put $\gamma=\frac{\beta+2}{\alpha+1}(>1)$ and let $\Omega_{\varepsilon}=\Omega \cap\{(x, y): y>\varepsilon\}$ for any $\varepsilon>0$.

Let $k \in \mathbb{N}$. By a classical compactness result in the unweighted case, there exists a function $u^{k} \in L^{1}\left(\Omega_{2^{-k}} ; y^{\beta}\right)$ and an increasing sequence of integers $\{a(k, m)\}_{m \geq 1}$ such that

$$
u_{a(k, m)} \rightarrow u^{k} \quad \text { in } L^{1}\left(\Omega_{2^{-k}} ; y^{\beta}\right) \text { and a.e. in } \Omega \text {. }
$$

By choosing $\{a(k+1, m)\}$ to be a subsequence of $\left\{a(k, m\},(k \in \mathbb{N})\right.$, we can achieve that $u^{k}=u^{k+1}$ in $\Omega_{2^{-k}}$, $k \in \mathbb{N}$.

Now put

$$
u(x)= \begin{cases}u^{1}(x) & \text { if } x \in \Omega_{2^{-1}} \\ u^{k}(x) & \text { if } x \in \Omega_{2^{-k}} \backslash \bar{\Omega}_{2^{-k+1}}, k=2,3, \ldots\end{cases}
$$

In view of our isoperimetric inequality, the sequence $u_{n}$ is equibounded in $L^{\gamma}\left(\Omega ; y^{\beta}\right)$. We have the following estimate:

$$
\begin{aligned}
\left.\|u\|_{L^{1}\left(\Omega_{2}-k\right.} ; y^{\beta}\right) & =\sum_{j=0}^{k} \int_{\Omega_{2-j} \backslash \bar{\Omega}_{2-j+1}}|u| y^{\beta} \mathrm{d} x \mathrm{~d} y \\
& \leq \sum_{j=0}^{k}\left(\int_{\Omega_{2^{-j}} \backslash \bar{\Omega}_{2-j+1}}|u|^{\gamma} y^{\beta} \mathrm{d} x \mathrm{~d} y\right)^{\frac{1}{\gamma}}\left(\int_{\Omega_{2-j} \backslash \bar{\Omega}_{2-j+1}} y^{\beta} \mathrm{d} x \mathrm{~d} y\right)^{1-\frac{1}{\gamma}}
\end{aligned}
$$




$$
\leq \sum_{j=0}^{k} C_{1}\left[\left.y^{\beta+1}\right|_{2^{-j}} ^{2^{-j+1}}\right]^{1-\frac{1}{\gamma}} \leq C_{2} \sum_{j=0}^{\infty} 2^{(-j+1)(\beta+1)\left(1-\frac{1}{\gamma}\right)}<+\infty
$$

with constants that do not depend on $k$. This implies that $u \in L^{1}\left(\Omega ; y^{\beta}\right)$.

Let $\varepsilon>0$. Choose $k$ large enough such that

$$
\int_{\Omega \backslash \bar{\Omega}_{2-k}} y^{\beta} \mathrm{d} x \mathrm{~d} y<\varepsilon
$$

and then $m$ large enough such that

$$
\int_{\Omega_{2-k}}\left|u-u_{a(k, m)}\right| y^{\beta} \mathrm{d} x \mathrm{~d} y<\varepsilon
$$

Then we obtain

$$
\begin{aligned}
\int_{\Omega}\left|u-u_{a(k, m)}\right| y^{\beta} \mathrm{d} x \mathrm{~d} y & <\varepsilon+\int_{\Omega \backslash \bar{\Omega}_{2-k}}\left|u-u_{a(k, m)}\right| y^{\beta} \mathrm{d} x \mathrm{~d} y \\
& \leq \varepsilon+\left(\int_{\Omega}\left|u-u_{a(k, m)}\right| y^{\beta} \mathrm{d} x \mathrm{~d} y\right)^{\frac{1}{\gamma}}\left(\int_{\Omega \backslash \bar{\Omega}_{2-k}} y^{\beta} \mathrm{d} x \mathrm{~d} y\right)^{1-\frac{1}{\gamma}} \\
& \leq \varepsilon+C \varepsilon^{\left(1-\frac{1}{\gamma}\right)(\beta+1)},
\end{aligned}
$$

where $C$ does not depend on $k$. From this the assertion follows.

Acknowledgements. We are grateful to M. van den Berg and to G. Csató for some inspiring conversations, and to V. Franceschi and G. Psaradakis for bringing some of their recent interesting results to our attention. The first, third, fourth and fifth authors are members of the Gruppo Nazionale per l'Analisi Matematica, la Probabilità e le loro Applicazioni (GNAMPA) of the Istituto Nazionale di Alta Matematica (INdAM) and they thank this institution for the support. The second author was partially supported by Leverhulme Trust ref. VP1-2017-004. The third author was partially supported by PRIN "Qualitative and quantitative aspects of nonlinear PDEs, 2017JPCAPN. We are also grateful to the Departments of Mathematics of Swansea University and of the University of Naples Federico II, and to South China University of Technology (ISAM) at Guangzhou for visiting appointments and their colleagues for their kind hospitality.

\section{REFERENCES}

[1] E. Abreu and L.G. Fernandes, On existence and nonexistence of isoperimetric inequalities with different monomial weights. Preprint arXiv:1904.01441v2 (2019).

[2] A. Alvino, F. Brock, F. Chiacchio, A. Mercaldo and M.R. Posteraro, Some isoperimetric inequalities on $\mathbb{R}^{N}$ with respect to weights $|x|^{\alpha}$. J. Math. Anal. Appl. 451 (2017) 280-318.

[3] A. Alvino, F. Brock, F. Chiacchio, A. Mercaldo and M.R. Posteraro, On weighted isoperimetric inequalities with non-radial densities. Appl. Anal. 98 (2019) 1935-1945.

[4] A. Alvino, F. Brock, F. Chiacchio, A. Mercaldo and M.R. Posteraro, The isoperimetric problem for a class of non-radial weights and applications. J. Differ. Equ. 267 (2019) 6831-6871.

[5] V. Bayle, A. Cañete, F. Morgan and C. Rosales, On the isoperimetric problem in Euclidean space with density. Calc. Var. PDE 31 (2008) 27-46.

[6] M.F. Betta, F. Brock, A. Mercaldo and M.R. Posteraro, A weighted isoperimetric inequality and applications to symmetrization. J. Inequal. Appl. 4 (1999) 215-240.

[7] M.F. Betta, F. Brock, A. Mercaldo and M.R. Posteraro, Weighted isoperimetric inequalities on $\mathbb{R}^{N}$ and applications to rearrangements. Math. Nachr. 281 (2008) 466-498.

[8] W. Boyer, B. Brown, G. Chambers, A. Loving and S. Tammen, Isoperimetric regions in $\mathbb{R}^{n}$ with density $r^{p}$. Anal. Geom. Metr. Spaces 4 (2016) 236-265.

[9] B. Brandolini, F. Della Pietra, C. Nitsch and C. Trombetti, Symmetry breaking in a constrained Cheeger type isoperimetric inequality. ESAIM: COCV 21 (2015) 359-371. 
[10] H. Brezis, Functional Analysis, Sobolev Spaces and Partial Differential Equations. Springer (2010).

[11] F. Brock, F. Chiacchio and A. Mercaldo, A weighted isoperimetric inequality in an orthant. Potential Anal. 41 (2012) 171-186.

[12] F. Brock, A. Mercaldo and M.R. Posteraro, On isoperimetric inequalities with respect to infinite measures. Rev. Mat. Iberoamericana 29 (2013) 665-690.

[13] D. Bucur and I. Fragalà, Proof of the honeycomb asymptotics for optimal Cheeger clusters. Adv. Math. 350 (2019) 97-129.

[14] D. Bucur and I. Fragalà, A Faber-Krahn inequality for the Cheeger constant of N-gons. J. Geom. Anal. 26 (2016) 88-117.

[15] X. Cabre and X. Ros-Oton, Sobolev and isoperimetric inequalities with monomial weights. J. Differ. Equ. 255 (2013) 43124336.

[16] X. Cabre, X. Ros-Oton and J. Serra, Euclidean balls solve some isoperimetric problems with nonradial weights. C. R. Math. Acad. Sci. Paris $\mathbf{3 5 0}$ (2012) 945-947.

[17] A. Cañete, M. Miranda Jr. and D. Vittone, Some isoperimetric problems in planes with density. J. Geom. Anal. 20 (2010) 243-290.

[18] T. Carroll, A. Jacob, C. Quinn and R. Walters, The isoperimetric problem on planes with density. Bull. Aust. Math. Soc. 78 (2008) 177-197.

[19] V. Caselles, M. Miranda jr and M. Novaga, Total variation and Cheeger sets in Gauss space. J. Funct. Anal. 259 (2010) $1491-1516$.

[20] H. Castro. Hardy-Sobolev inequalities with monomial weights. Ann. Mat. Pura Appl. 196 (2017) 579-598.

[21] G.R. Chambers, Proof of the Log-Convex Density Conjecture. J. Eur. Math. Soc. 21 (2019) 2301-2332.

[22] J. Cheeger, A lower bound for the smallest eigenvalue of the Laplacian. Problems in analysis: A symposium in honor of Salomon Bochner (1970) 195-199.

[23] G. Csató, An isoperimetric problem with density and the Hardy Sobolev inequality in $\mathbb{R}^{2}$. Differ. Int. Equ. 28 (2015) 971-988.

[24] J. Dahlberg, A. Dubbs, E. Newkirk and H. Tran, Isoperimetric regions in the plane with density $r^{p}$. New York J. Math. 16 (2010) 31-51.

[25] G. De Philippis, G. Franzina and A. Pratelli, Existence of isoperimetric sets with densities "converging from below" on $\mathbb{R}^{N}$. J. Geom. Anal. 27 (2017) 1086-1105.

[26] L. Di Giosia, J. Habib, L. Kenigsberg, D. Pittman and W. Zhu, Balls Isoperimetric in $\mathbb{R}^{n}$ with Volume and Perimeter Densities $r^{m}$ and $r^{k}$. Preprint arXiv:1610.05830v2 (2019).

[27] A. Diaz, N. Harman, S. Howe and D. Thompson, Isoperimetric problems in sectors with density. Adv. Geom. 12 (2012) 589-619.

[28] V. Franceschi, A minimal partition problem with trace constraint in the Grushin plane. Calc. Var. Partial Differ. Equ. 56 (2017) 104

[29] V. Franceschi and R. Monti, Isoperimetric problem in H-type groups and Grushin spaces. Rev. Mat. Iberoam. 32 (2016) $1227-1258$.

[30] V. Franceschi and G. Stefani, Symmetric double bubbles in the Grushin plane. ESAIM: COCV 25 (2019) 37.

[31] P. Gurka and B. Opic, Continuous and compact imbeddings of weighted Sobolev spaces II. Czechoslovak Math. J. 39 (1989) 78-94.

[32] N. Harman, S. Howe and F. Morgan, Steiner and Schwarz symmetrization in warped products and fiber bundles with density. Rev. Mat. Iberoamericana 27 (2011) 909-918.

[33] S. Howe, The Log-Convex Density Conjecture and vertical surface area in warped products. Adv. Geom. 15 (2015) 455-468.

[34] I.R. Ionescu and T. Lachand-Robert, Generalized Cheeger sets related to landslides. Calc. Var. Partial Differ. Equ. 23 (2005) $227-249$.

[35] B. Kawohl and V. Fridman, Isoperimetric estimates for the first eigenvalue of the p-Laplace operator and the Cheeger constant. Comment. Math. Univ. Carolin. 44 (2003) 659-667.

[36] A.V. Kolesnikov and R.I. Zhdanov, On isoperimetric sets of radially symmetric measures. Concentration, functional inequalities and isoperimetry In Vol. 545 of Contemp. Math. Amer. Math. Soc., Providence, RI (2011) 123-154.

[37] C. Maderna and S. Salsa. Sharp estimates for solutions to a certain type of singular elliptic boundary value problems in two dimensions. Applicable Analysis 12 (1981) 307-321.

[38] V. Maz'ja, Lectures on isoperimetric and isocapacitary inequalities in the theory of Sobolev spaces. Heat kernels and analysis on manifolds, graphs, and metric spaces (Paris, 2002). In Vol. 338 of Contemp. Math. Amer. Math. Soc., Providence, RI (2003) $307-340$.

[39] V. Maz'ja and T. Shaposhnikova, A collection of sharp dilation invariant integral inequalities for differentiable functions. Sobolev spaces in mathematics I. In Vol. 8 of Int. Math. Ser. (N.Y.). Springer (2009) 223-247.

[40] R. Monti and D. Morbidelli, Isoperimetric inequality in the Grushin plane. J. Geom. Anal. 14 (2004) $355-368$.

[41] F. Morgan, Manifolds with density. Notices Amer. Math. Soc. 52 (2005) 853-858.

[42] F. Morgan, The Log-Convex Density Conjecture. Contemp. Math. 545 (2011) 209-211.

[43] F. Morgan and A. Pratelli, Existence of isoperimetric regions in $\mathbb{R}^{N}$ with density. Ann. Global Anal. Geom. 43 (2013) 331-365.

[44] E. Parini, An introduction to the Cheeger problem. Surv. Math. Appl. 6 (2011) 9-21.

[45] A. Pratelli and G. Saracco, On the isoperimetric problem with double density. Nonlinear Anal. 177 (2018) $733-752$.

[46] A. Pratelli and G. Saracco, The $\varepsilon-\varepsilon^{\beta}$ property in the isoperimetric problem with double density, and the regularity of isoperimetric sets. Adv. Nonlinear Stud. 20 (2020) 539-555.

[47] G. Saracco, Weighted Cheeger sets are domains of isoperimetry. Manuscripta Math. 156 (2018) 371-381. 